\title{
WEIGHT TWO MOTIVIC COHOMOLOGY OF CLASSIFYING SPACES FOR SEMISIMPLE GROUPS
}

\author{
A. MERKURJEV
}

\begin{abstract}
Let $f: X \rightarrow Y$ be a torsor for a semisimple group $G$ with $Y$ a smooth and geometrically irreducible variety over an arbitrary field. We relate the étale motivic cohomology of weight two for $X, Y$ and $G$. We also compute the étale motivic cohomology groups of degree at most 4 for the classifying space of $G$.
\end{abstract}

\section{INTRODUCTION}

Let $G$ be a smooth algebraic group over a field $F$ and $f: X \rightarrow Y$ a $G$ torsor with $Y$ smooth and geometrically irreducible over $F$. In [14], Sansuc constructed an exact sequence

$$
\begin{aligned}
1 \longrightarrow F[Y]^{\times} \longrightarrow F[X]^{\times} \longrightarrow \widehat{G} \longrightarrow \operatorname{Pic}(Y) \longrightarrow & \\
& \operatorname{Pic}(X) \longrightarrow \operatorname{Pic}(G) \longrightarrow \operatorname{Br}(Y) \longrightarrow \operatorname{Br}(X),
\end{aligned}
$$

where $\widehat{G}=\operatorname{Hom}\left(G, \mathbb{G}_{m}\right)$ is the character group of $G$, Pic and Br are the Picard and the (cohomological) Brauer group, respectively. The exact sequence can be viewed as a relation between the étale cohomology groups of $Y, X$ and $G$ with values in the multiplicative sheaf $\mathbb{G}_{m}$ since for every variety $U$ over $F$, we have

$$
H^{i}\left(U, \mathbb{G}_{m}\right)= \begin{cases}F[U]^{\times}, & \text {if } i=0 ; \\ \operatorname{Pic}(U), & \text { if } i=1 ; \\ \operatorname{Br}(U), & \text { if } i=2 .\end{cases}
$$

Recall that the sheaf $\mathbb{G}_{m}$ is the motivic sheaf $\mathbb{Z}(1)$ shifted by 1 and thus the groups above are the étale motivic cohomology groups of weight 1 (with values in $\mathbb{Z}(1)$ ).

In the present paper, we extend Sansuc's result to the weight two étale motivic cohomology (with values in $\mathbb{Z}(2)$ ) in the case $G$ is a semisimple group (Theorem 5.1). We adopt the definition of $\mathbb{Z}(2)$ given in [8] and [9].

In Theorem 5.3, we compute the groups $H^{i}(B G, \mathbb{Z}(2))$ for $i \leq 4$, where $B G$ is the classifying space of the group $G$. This result was used in [10] for the

Date: February, 2013.

Key words and phrases. Semisimple groups, torsors, motivic cohomology, classifying space 2010 Mathematical Subject Classifications: 14F42, 14L30, 14 M1\%.

The work has been supported by the NSF grant DMS \#1160206. 
computation of the group of degree 3 cohomological invariants of semisimple groups with coefficients in $\mathbb{Q} / \mathbb{Z}(2)$.

In the proof of Theorem 5.1, following [4, Lemma B.2], we introduce the relative motivic complex $\mathbb{Z}_{f}(2)$ for a $G$-torsor $f: X \rightarrow Y$ that connects the weight two motivic cohomology of $X$ and $Y$. We construct the exact triangle (4.12) containing $\mathbb{Z}_{f}(2)$ in the derived category of etale sheaves on $Y$. The other terms of the exact triangle are determined by the combinatorics of $G$ (the root system, weight and root lattices). Some verifications are lengthy. For the reader's convenience, we move the technical computations to the Appendix.

We don't impose any characteristic assumption in the paper. Sometimes we need to treat the "bad" characteristic case separately, but the main results are stated in a uniform way.

Acknowledgements: I would like to thank Paul Balmer and Bruno Kahn for helpful discussions and comments.

1a. Notation. We will use the following notation in the paper.

$F$ is the base field (of arbitrary characteristic),

$F_{\text {sep }}$ a separable closure of $F$,

$\Gamma_{F}=\operatorname{Gal}\left(F_{\text {sep }} / F\right)$,

$K_{*}$ and $K_{*}^{Q}$ denote Milnor's and Quillen's $K$-groups, respectively.

We identify (continuous) Galois modules (i.e., $\Gamma_{F}$-modules) and étale sheaves of abelian groups on Spec $F$. For two sheaves $A$ and $B$, we write $A * B$ for the sheaf corresponding to the Galois module $\operatorname{Tor}_{1}^{\mathbb{Z}}\left(A\left(F_{\text {sep }}\right), B\left(F_{\text {sep }}\right)\right)$.

For a complex $A$ of étale sheaves on a variety $X$, we write $H^{*}(X, A)$ for the étale (hyper-)cohomology group of $X$ with values in $A$.

Let $X$ be an algebraic variety over $F$. For any $i \geq 0$, the homology group of the complex $C^{\bullet}\left(X, K_{d}\right)$ :

$\ldots \longrightarrow \coprod_{x \in X^{(i-1)}} K_{d-i+1}(F(x)) \stackrel{\partial}{\longrightarrow} \coprod_{x \in X^{(i)}} K_{d-i}(F(x)) \stackrel{\partial}{\longrightarrow} \coprod_{x \in X^{(i+1)}} K_{d-i-1}(F(x)) \longrightarrow \ldots$,

where $X^{(i)}$ is the set of point in $X$ of codimension $i$, will be denoted by $A^{i}\left(X, K_{d}\right)$ (see [13]). In particular, $A^{i}\left(X, K_{i}\right)=\mathrm{CH}^{i}(X)$ is the Chow group of classes of algebraic cycles on $X$ of codimension $i$.

\section{INVARIANT QUADRATIC FORMS}

Let $R$ be a root system. Write $\Lambda_{r} \subset \Lambda_{w}$ for the root and the weight lattices respectively. The goal of this section is to introduce an abelian group $D(R, \Lambda)$ associated with the root system $R$ and an intermediate lattice $\Lambda$ between $\Lambda_{r}$ and $\Lambda_{w}$.

Let $R^{*}$ be the dual root system. The lattices $\Lambda_{r}^{*}$ and $\Lambda_{w}^{*}$ are dual to $\Lambda_{w}$ and $\Lambda_{r}$, respectively. Choose a system of simple roots $\alpha_{1}, \ldots \alpha_{n}$; they form a $\mathbb{Z}$-basis for $\Lambda_{r}$. The dual system of simple co-roots $\alpha_{1}^{*}, \ldots \alpha_{n}^{*}$ is a basis for $\Lambda_{r}^{*}$. The fundamental weights $f_{1}, \ldots, f_{n}$ form the basis for $\Lambda_{w}$ dual to the basis of simple co-roots. Similarly, write $f_{1}^{*}, \ldots, f_{n}^{*}$ for the fundamental co-weights. Write $W$ for the Weyl group of both $R$ and $R^{*}$. 
If $R$ is irreducible, there is a unique $W$-invariant integral quadratic form $q$ on $\Lambda_{r}^{*}$ having value 1 on all short co-roots of $R^{*}$. We view $q$ as an element of the symmetric square $S^{2}\left(\Lambda_{w}\right)$. In fact, $q$ generates the group $S^{2}\left(\Lambda_{w}\right)^{W}$ of $W$-invariant elements in $S^{2}\left(\Lambda_{w}\right)$ (see [5, Part II, $\left.\S 7\right]$ ). Write $b_{q}$ for the polar form of $q$, so $b_{q}$ is an integral symmetric bilinear form on $\Lambda_{r}^{*}$ defined by $b_{q}(x, y)=q(x+y)-q(x)-q(y)$. Thus, $b_{q}$ can be viewed as an element of $\Lambda_{w} \otimes \Lambda_{w}$ in the image of the map

$$
S^{2}\left(\Lambda_{w}\right) \longrightarrow \Lambda_{w} \otimes \Lambda_{w}, \quad x y \mapsto x \otimes y+y \otimes x .
$$

Lemma 2.1. The bilinear form $b_{q}$ extends to a bilinear map $\Lambda_{r}^{*} \times \Lambda_{w}^{*} \rightarrow \mathbb{Z}$. In particular, $b_{q} \in \Lambda_{w} \otimes \Lambda_{r}$.

Proof. The form $b_{q}$ extends uniquely to a $W$-invariant bilinear map $b_{q}^{\prime}: \Lambda_{r}^{*} \times$ $\Lambda_{w}^{*} \longrightarrow \mathbb{Q}$. It suffices to show that $b_{q}^{\prime}\left(\alpha_{i}^{*}, f_{j}^{*}\right) \in \mathbb{Z}$ for all $i$ and $j$. Let $s_{i}$ be the reflection in $\Lambda_{w}^{*}$ with respect to $\alpha_{i}^{*}$. We have $s_{i}\left(\alpha_{i}^{*}\right)=-\alpha_{i}^{*}$ and $s_{i}\left(f_{j}^{*}\right)=$ $f_{j}^{*}-\delta_{i j} \alpha_{i}^{*}$. As $b_{q}^{\prime}$ is $W$-invariant, we have

$$
b_{q}^{\prime}\left(\alpha_{i}^{*}, f_{j}^{*}\right)=b_{q}^{\prime}\left(s_{i}\left(\alpha_{i}^{*}\right), s_{i}\left(f_{j}^{*}\right)\right)=b_{q}^{\prime}\left(-\alpha_{i}^{*}, f_{j}^{*}-\delta_{i j} \alpha_{i}^{*}\right),
$$

therefore, $b_{q}^{\prime}\left(\alpha_{i}^{*}, f_{j}^{*}\right)=\delta_{i j} b_{q}\left(\alpha_{i}^{*}, \alpha_{i}^{*}\right) / 2=\delta_{i j} q\left(\alpha_{i}^{*}\right) \in \mathbb{Z}$.

In the general case, when $R$ is not necessarily irreducible, the group $S^{2}\left(\Lambda_{w}\right)^{W}$ has the canonical basis $\left\{q_{1}, q_{2}, \ldots, q_{s}\right\}$, where $q_{i}$ are the canonical quadratic forms as above corresponding to the connected components of the root system. Lemma 2.1 yields then the following proposition.

Proposition 2.2. For every root system, the image of $S^{2}\left(\Lambda_{w}\right)^{W}$ under the map (2.1) is contained in $\Lambda_{w} \otimes \Lambda_{r}$.

Choose a lattice $\Lambda$ such that $\Lambda_{r} \subset \Lambda \subset \Lambda_{w}$. Note that as $W$ acts trivially on $\Lambda_{w} / \Lambda_{r}$, the lattice $\Lambda$ is $W$-invariant.

Set $\Delta:=\Lambda_{w} / \Lambda$, and consider the inclusion homomorphism $i: \Lambda \rightarrow \Lambda_{w}$ and the canonical homomorphism $p: \Lambda_{w} \rightarrow \Delta$. Consider also the commutative diagram

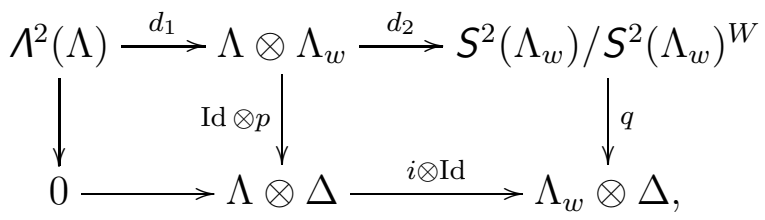

where $d_{1}$ takes $x \wedge y$ to $x \otimes y-y \otimes x, d_{2}$ is induced by the canonical map $\Lambda_{w} \otimes \Lambda_{w} \rightarrow S^{2}\left(\Lambda_{w}\right)$ and $q$ takes the class of $x y$ to $x \otimes p(y)+y \otimes p(x)$. Note that $q$ is well defined by Proposition 2.2 since $\Lambda_{r} \subset \Lambda=\operatorname{Ker}(p)$.

Write $D(R, \Lambda)$ for the homology group of top row of the diagram (2.2). The diagram yields a canonical homomorphism

$$
\kappa: D(R, \Lambda) \longrightarrow \Delta * \Delta:=\operatorname{Tor}_{1}^{\mathbb{Z}}(\Delta, \Delta)=\operatorname{Ker}(i \otimes \mathrm{Id}) .
$$

The kernel $Q(R, \Lambda)$ of $\kappa$ coincides with $S^{2}(\Lambda) \cap S^{2}\left(\Lambda_{w}\right)^{W}=S^{2}(\Lambda)^{W}$. Thus, $Q(R, \Lambda)$ is a lattice of the rank the number of connected components of $R$. 


\section{The groups $A^{0}\left(X, K_{2}\right)$ And $A^{1}\left(X, K_{2}\right)$}

In this section $G$ is a semisimple group over a field $F$ and $C$ is the kernel of a simply connected cover $G^{\mathrm{sc}} \rightarrow G$. By $(1.1), \widehat{C}=\operatorname{Pic}(G)=\mathrm{CH}^{1}(G)$.

3a. K-cohomology of semisimple groups. We generalize the approach given in [5, Part II, §6] for simply connected groups to arbitrary semisimple groups.

Let $G$ be a split semisimple group and $T \subset G$ a split maximal torus. Let $G^{\text {sc }} \rightarrow G$ be a simply connected cover and $T^{\text {sc }}$ the maximal torus of $G^{\text {sc }}$ over $T$.

Set $Z:=G / T=G^{\mathrm{sc}} / T^{\mathrm{sc}}$. By [5, Part II, $\left.\S 6\right]$, there is a canonical isomorphism $\mathrm{CH}^{1}(Z) \simeq \widehat{T}^{\mathrm{sc}}$ and the kernel of the surjective product homomorphism

$$
S^{2}\left(\widehat{T}^{\mathrm{sc}}\right) \longrightarrow \mathrm{CH}^{2}(Z)
$$

coincides with $S^{2}\left(\widehat{T}^{\mathrm{sc}}\right)^{W}$, where $W$ is the Weyl groups of $G$.

The fiber of the natural morphism $\pi: G \rightarrow Z$ is a torsor for the split torus $T$, and hence is isomorphic to $T$. In follows that in the spectral sequence $[13$, $\S 8]$

$$
E_{1}^{p, q}=\coprod_{z \in Z^{(p)}} A^{q}\left(\pi^{-1}(z), K_{n-p}\right) \Longrightarrow A^{p+q}\left(G, K_{n}\right)
$$

we have $E_{1}^{p, q}=0$ if $q>0$ by [5, Part II, Proposition 5.6.2]. It follows that

$$
A^{p}\left(G, K_{n}\right) \simeq A^{p}\left(Z, M_{n}\right),
$$

where $M$ is a cycle module over $Z$ defined by

$$
M_{n}(z)=A^{0}\left(\pi^{-1} z, K_{n}\right)
$$

for every point $z \in Z(K)$ for a field extension $K / F$.

By [5, Part II, §5.7], there is a filtration by cycle modules

$$
0=M_{n}^{(1)} \subset M_{n}^{(0)} \subset \ldots M_{n}^{(1-n)} \subset M_{n}^{(-n)}=M_{n}
$$

with the factor cycle modules

$$
M_{n}^{(p / p+1)} \simeq \Lambda^{-p}(\widehat{T}) \otimes K_{n+p} .
$$

The $E_{1}$-term of the spectral sequence

$$
E_{1}^{p, q}=A^{p+q}\left(Z, M_{n}^{(p / p+1)}\right) \Longrightarrow A^{p+q}\left(Z, M_{n}\right),
$$

associated with the filtration is isomorphic to

$$
\Lambda^{-p}(\widehat{T}) \otimes A^{p+q}\left(Z, K_{n+p}\right) \simeq \Lambda^{-p}(\widehat{T}) \otimes \mathrm{CH}^{p+q}(Z) \otimes K_{n-q}(F)
$$

by [5, Part II, Lemma 6.5]. Thus, we have a spectral sequence

$$
E_{1}^{p, q}=\Lambda^{-p}(\widehat{T}) \otimes \mathrm{CH}^{p+q}(Z) \otimes K_{n-q}(F) \Longrightarrow A^{p+q}\left(G, K_{n}\right) .
$$

When $n=2$, the nontrivial $E_{1}$-terms of the spectral sequence are the following: 


$$
\begin{gathered}
\Lambda^{2}(\widehat{T}) \stackrel{d_{1}}{\longrightarrow} \widehat{T} \otimes \widehat{T}^{\mathrm{sc}} \stackrel{d_{2}}{\longrightarrow} S^{2}\left(\widehat{T}^{\mathrm{sc}}\right) / S^{2}\left(\widehat{T}^{\mathrm{sc}}\right)^{W} \\
\widehat{T} \otimes F^{\times} \stackrel{i \otimes \mathrm{Id}}{\longrightarrow} \widehat{T}^{\mathrm{sc}} \otimes F^{\times} \\
K_{2}(F) .
\end{gathered}
$$

The differentials of the similar spectral sequence for the natural morphism $G^{\text {sc }} \rightarrow Z$ were computed in [5, Part II, §6]. As the two spectral sequences are related by the pull-back homomorphisms with respect to the morphisms $G^{\text {sc }} \rightarrow G$ and $T^{\text {sc }} \rightarrow T$, the computation of the differentials shows that the top row of the diagram coincides with the top row of the diagram (2.2) with $R$ the root system of $G$ relative to the torus $T$, the weight lattice $\Lambda_{w}=\widehat{T}^{\mathrm{sc}}$, the lattice $\Lambda=\widehat{T}$ and $\Delta=\widehat{C}$, where

$$
C:=\operatorname{Ker}\left(T^{\mathrm{sc}} \rightarrow T\right)=\operatorname{Ker}\left(G^{\mathrm{sc}} \rightarrow G\right) .
$$

Write $D(G)$ for the group $D(R, \Lambda)$ defined in Section 2. Note that $D(G)$ does not change under field extensions, i.e., for any field extension $K / F$, the natural homomorphism $D(G) \rightarrow D\left(G_{K}\right)$ is an isomorphism.

Recall that we have a natural homomorphism

$$
\kappa: D(G) \longrightarrow \widehat{C} * \widehat{C} .
$$

Write $Q(G)$ for the lattice

$$
Q(R, \Lambda)=\operatorname{Ker}(\kappa)=S^{2}(\widehat{T})^{W} .
$$

Remark 3.1. The group $Q(G)$ for a split semisimple group $G$ has intrinsic description as the group of all quadratic integral-valued functions on the set of all loops in $G$ (see $[7, \S 31]$ and [5, Part II, §7]).

In the spectral sequence the map $d_{1}$ is injective and

$$
\operatorname{Ker}(i \otimes \mathrm{Id})=\operatorname{Tor}_{1}^{\mathbb{Z}}\left(\widehat{C}, F^{\times}\right)=\widehat{C} * F^{\times}=\widehat{C} * \mu(F),
$$

where $\mu(F)$ is the group of roots of unity in $F$. The spectral sequence then yields the following proposition.

Proposition 3.2. Let $G$ be a split semisimple group over a field $F$. Then there are natural exact sequences

$$
\begin{aligned}
& 0 \longrightarrow K_{2}(F) \longrightarrow A^{0}\left(G, K_{2}\right) \longrightarrow \widehat{C} * \mu(F) \longrightarrow 0, \\
& 0 \longrightarrow \widehat{C} \otimes F^{\times} \longrightarrow A^{1}\left(G, K_{2}\right) \longrightarrow D(G) \longrightarrow 0 .
\end{aligned}
$$


3b. The groups $D(G)$ and $Q(G)$. Let $G$ be an arbitrary (not necessarily split) semisimple group over $F$. Then $G_{\text {sep }}$ is split and $D\left(G_{\text {sep }}\right)$ is a Galois module over $F$, i.e., a (continuous) $\Gamma_{F}$-module, where $\Gamma_{F}=\operatorname{Gal}\left(F_{\text {sep }} / F\right)$. Write $D(G)$ for the corresponding étale sheaf over $F$. In particular, $D(G)(F)=$ $D\left(G_{\text {sep }}\right)^{\Gamma_{F}}$. We will simply write $D(G)$ for $D(G)(F)$ and $Q(G)$ for the kernel of $\kappa: D(G) \rightarrow(\widehat{C} * \widehat{C})(F)$.

For a field extension $L / F$, write $\widehat{C}(L)$ for the character group of $C$ over $L$. Denote by $\widehat{C}$ the étale sheaf over $F$ corresponding to the Galois module $\widehat{C}\left(F_{\text {sep }}\right)$. In particular, $\widehat{C}(F)=\widehat{C}\left(F_{\text {sep }}\right)^{\Gamma_{F}}$.

Write $G^{\mathrm{qs}}$ for the quasisplit twisted form of $G$ (see [16]). Thus, $G^{\mathrm{qs}}$ is an inner twisted form of $G$. In particular, $G_{\mathrm{sep}}^{\mathrm{qs}} \simeq G_{\mathrm{sep}}$. Let $T^{\mathrm{qs}}$ be the maximal torus of $G_{\mathrm{sep}}^{\mathrm{qs}}$ corresponding to $T_{\mathrm{sep}}$ under this isomorphism and $T^{\mathrm{qcqs}}$ the inverse image of $T^{\mathrm{qs}}$ under the simply connected quasisplit cover of $G_{\mathrm{sep}}^{\mathrm{qs}}$. The character lattice $\widehat{T}^{\text {qcqs }}$ is spanned by the fundamental weights and the Galois group $\Gamma_{F}$ permutes them according to the action on the Dynkin diagram of $G$ (the socalled $*$-action, see $[16]$ ).

For every variety $X$ over $F$ define $D\left(X_{\text {sep }}\right)$ as the cokernel of the product homomorphism

$$
\mathrm{CH}^{1}\left(X_{\text {sep }}\right) \otimes F_{\text {sep }}^{\times} \longrightarrow A^{1}\left(X_{\text {sep }}, K_{2}\right) .
$$

By Proposition 3.2, the group $D\left(G_{\text {sep }}\right)$ for a split semisimple group $G$ coincides with the one defined previously.

Proposition 3.3. Let $X \rightarrow \operatorname{Spec} F$ be a G-torsor. Then the Galois modules $D\left(X_{\mathrm{sep}}\right), D\left(G_{\mathrm{sep}}\right)$ and $D\left(G_{\mathrm{sep}}^{\mathrm{qs}}\right)$ are all canonically isomorphic.

Proof. The Galois actions on $G_{\text {sep }}$ and $G_{\text {sep }}^{\text {qs }}$ differ by the conjugation action of $\bar{G}\left(F_{\text {sep }}\right)$, where $\bar{G}$ is the corresponding adjoint group. Similarly, the Galois actions on $X_{\text {sep }}$ and $G_{\text {sep }}$ differ by an action of $G\left(F_{\text {sep }}\right)$. The statement follows from Lemma 6.2 applied to the homotopy invariant functor $D$ (see Example $6.1)$ as $D\left(G_{\text {sep }}\right)$ does not change under field extensions.

3c. The group $A^{0}\left(X, K_{2}\right)$. For a variety $X$ over $F$, write $\bar{A}^{0}\left(X, K_{2}\right)$ for the cokernel of the natural homomorphism $K_{2}(F) \rightarrow A^{0}\left(X, K_{2}\right)$.

As $G$ is smooth, by [15, Corollary 5.9] and Proposition 3.2, there are natural isomorphisms

$$
\bar{A}^{0}\left(G, K_{2}\right) \simeq \bar{A}^{0}\left(G_{\mathrm{sep}}, K_{2}\right)^{\Gamma_{F}} \simeq(\widehat{C} * \mu)(F),
$$

where $\widehat{C}$ and $\mu$ are viewed as locally constant étale sheaves.

In particular, if $K / F$ is a field extension such that $F$ is separably closed in $K$, then the natural homomorphism

$$
\bar{A}^{0}\left(G, K_{2}\right) \longrightarrow \bar{A}^{0}\left(G_{K}, K_{2}\right)
$$

is an isomorphism. By Lemma 6.2, the natural action of $G(F)$ on $\bar{A}^{0}\left(G, K_{2}\right)$ is trivial. 
Proposition 3.4. Let $f: X \rightarrow Y$ be a trivial $G$-torsor with $Y$ a geometrically irreducible variety over $F$. Then there is a natural split exact sequence

$$
0 \longrightarrow A^{0}\left(Y, K_{2}\right) \longrightarrow A^{0}\left(X, K_{2}\right) \longrightarrow(\widehat{C} * \mu)(F) \longrightarrow 0 .
$$

Proof. The Rost spectral sequence $[13, \S 8]$ for $f$ yields the exactness of the middle row in the diagram

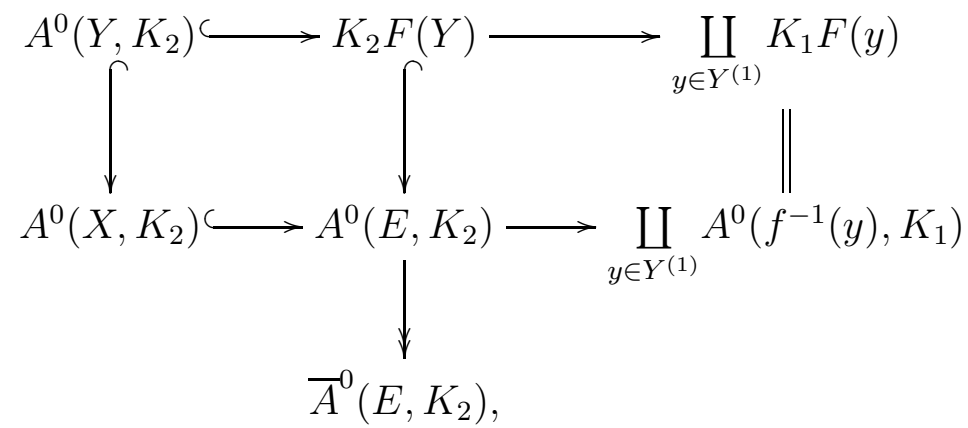

where $E$ is the generic fiber of $f$. Note that $E$ is isomorphic to $G_{F(Y)}$ and the induced isomorphism $\bar{A}^{0}\left(E, K_{2}\right) \simeq \bar{A}^{0}\left(G_{F(Y)}, K_{2}\right)$ is independent of the isomorphism between $E$ and $G_{F(Y)}$ as the group of $F(Y)$-points of $G$ acts trivially on $\bar{A}^{0}\left(G_{F(Y)}, K_{2}\right) \simeq(\widehat{C} * \mu)(F)$. Also, the later group is canonically isomorphic to $\vec{A}^{0}\left(G, K_{2}\right)$ since $F$ is algebraically closed in $F(Y)$.

This proves the exactness at the first and the middle terms of the sequence. In fact, the sequence is split by the homomorphism $A^{0}\left(G, K_{2}\right) \rightarrow A^{0}\left(X, K_{2}\right)$ induced by the composition $X \stackrel{\sim}{\rightarrow} G \times Y \rightarrow G$.

Let $X \rightarrow Y$ be a (not necessarily trivial) $G$-torsor with $Y$ a geometrically irreducible variety over $F$. Then the first projection $p: X \times_{Y} X \rightarrow X$ is a trivial $G$-torsor and by Proposition 3.4, we have the composition

$$
\alpha: A^{0}\left(X, K_{2}\right) \stackrel{p^{*}}{\longrightarrow} A^{0}\left(X \times_{Y} X, K_{2}\right) \longrightarrow(\widehat{C} * \mu)(F) .
$$

3 d. The group $A^{1}\left(X, K_{2}\right)$. Let $f: X \rightarrow Y$ be a trivial $G$-torsor over a geometrically irreducible variety $Y$ over $F$ with $G$ split. The nontrivial $E_{1}$ terms of the Rost spectral sequence for $f[13, \S 8]$ :

$$
E_{1}^{p, q}=\coprod_{y \in Y^{(p)}} A^{q}\left(f^{-1}(y), K_{2-p}\right) \Longrightarrow A^{p+q}\left(X, K_{2}\right)
$$

are the following:

$$
\begin{aligned}
& A^{1}\left(E, K_{2}\right) \stackrel{d}{\longrightarrow} \coprod_{y \in Y^{(1)}} A^{1}\left(f^{-1}(y), K_{1}\right) \\
& A^{0}\left(E, K_{2}\right) \longrightarrow \coprod_{y \in Y^{(1)}} A^{0}\left(f^{-1}(y), K_{1}\right) \longrightarrow \coprod_{y \in Y^{(2)}} A^{0}\left(f^{-1}(y), K_{0}\right),
\end{aligned}
$$


where $E$ is the generic fiber of $f$.

Note that $A^{0}\left(f^{-1}(y), K_{i}\right)=K_{i} F(y)$ for $i=0$ or 1 , and $A^{1}\left(f^{-1}(y), K_{1}\right)=$ $\mathrm{CH}^{1}\left(G_{y}\right)=\widehat{C}$. We then have an exact sequence

$$
A^{1}\left(Y, K_{2}\right) \longrightarrow A^{1}\left(X, K_{2}\right) \longrightarrow \operatorname{Ker}(d) \longrightarrow \mathrm{CH}^{2}(Y) \longrightarrow \mathrm{CH}^{2}(X) .
$$

As $f$ is a trivial $G$-torsor, the first and the last homomorphisms in the sequence are injective, hence $A^{1}\left(Y, K_{2}\right)$ can be viewed as a subgroup of $A^{1}\left(X, K_{2}\right)$ and we have an isomorphism

$$
A^{1}\left(X, K_{2}\right) / A^{1}\left(Y, K_{2}\right) \simeq \operatorname{Ker}(d) .
$$

By Proposition 3.2, there is a natural surjective homomorphism

$$
A^{1}\left(E, K_{2}\right) \rightarrow D\left(G_{F(Y)}\right)=D(G)
$$

with the kernel $\widehat{C} \otimes F(Y)^{\times}$. The commutative diagram

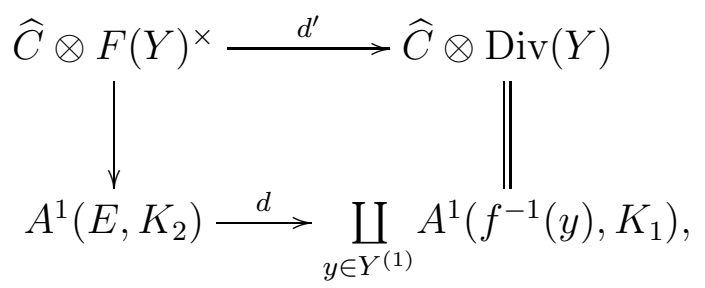

where Div is the divisor group and $d^{\prime}=1_{\widehat{C}} \otimes$ div, yields an exact sequence

$$
0 \longrightarrow \operatorname{Ker}\left(d^{\prime}\right) \longrightarrow \operatorname{Ker}(d) \stackrel{\gamma}{\longrightarrow} D(G) \text {. }
$$

The last homomorphism in the sequence is surjective as it is split by the homomorphism $A^{1}\left(G, K_{2}\right) \rightarrow A^{1}\left(E, K_{2}\right)$ induced by the composition $E \stackrel{\sim}{\rightarrow}$ $G_{F(Y)} \rightarrow G$.

As the group $\operatorname{PDiv}(Y)$ of principal divisors on $Y$ is torsion-free, we have the following diagram with the exact column and row:

$$
\begin{aligned}
& \widehat{C} * \mathrm{CH}^{1}(Y) \\
& \downarrow \\
& 0 \longrightarrow \widehat{C} \otimes A^{0}\left(Y, K_{1}\right) \longrightarrow \widehat{C} \otimes F(Y)^{\times} \longrightarrow \widehat{C} \otimes \operatorname{PDiv}(Y) \longrightarrow 0
\end{aligned}
$$

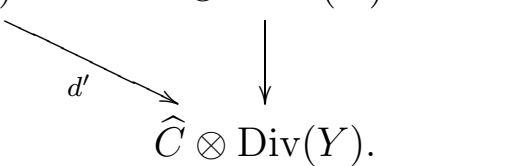

Therefore, there is an exact sequence

$$
0 \longrightarrow \widehat{C} \otimes A^{0}\left(Y, K_{1}\right) \longrightarrow \operatorname{Ker}\left(d^{\prime}\right) \longrightarrow \widehat{C} * \mathrm{CH}^{1}(Y) \longrightarrow 0 .
$$

We have proved:

Proposition 3.5. Let $X \rightarrow Y$ be a trivial $G$-torsor with $Y$ a geometrically irreducible variety over $F$ and $G$ split. Then the sequence

$$
0 \longrightarrow \widehat{C} \otimes A^{0}\left(Y, K_{1}\right) \longrightarrow A^{1}\left(X, K_{2}\right) / A^{1}\left(Y, K_{2}\right) \stackrel{\gamma}{\longrightarrow} D(G) \longrightarrow 0
$$


is exact, except at the middle term, where the homology group is naturally isomorphic to $\widehat{C} * \mathrm{CH}^{1}(Y)$.

Corollary 3.6. Let $X \rightarrow \operatorname{Spec} F$ be a trivial $G$-torsor with $G$ split. Then the sequence

$$
0 \longrightarrow \widehat{C} \otimes F^{\times} \longrightarrow A^{1}\left(X, K_{2}\right) \stackrel{\gamma}{\longrightarrow} D(G) \longrightarrow 0
$$

is exact.

Let $X \rightarrow Y$ be a (not necessarily trivial) $G$-torsor with $Y$ a geometrically irreducible variety over $F$. Then the first projection $X \times_{Y} X \rightarrow X$ is a trivial $G$-torsor. As $G$ is split over $F_{\text {sep }}$, by Proposition 3.5 we have the composition

$$
\gamma: A^{1}\left(X, K_{2}\right) \longrightarrow A^{1}\left(X_{\mathrm{sep}} \times_{Y_{\mathrm{sep}}} X_{\mathrm{sep}}, K_{2}\right)^{\Gamma_{F}} \longrightarrow D\left(G_{\mathrm{sep}}\right)^{\Gamma_{F}}=D(G) .
$$

\section{The COMPLEXES $\mathbb{Z}_{f}(i)$}

For a smooth variety $X$ over $F$ we write $\mathbb{Z}_{X}(i)$ with $i=1$ or 2 for the motivic complex of étale sheaves defined in [8] and [9]. By [6, Theorem 1.1], we have the following formulas for the étale motivic cohomology of weight 1 and 2 :

$$
\begin{aligned}
& H^{q}\left(X, \mathbb{Z}_{X}(1)\right)= \begin{cases}0, & \text { if } q \leq 0 ; \\
A^{0}\left(X, K_{1}\right)=F[X]^{\times}, & \text {if } q=1 ; \\
A^{1}\left(X, K_{1}\right)=\mathrm{CH}^{1}(X), & \text { if } q=2 ; \\
\operatorname{Br}(X), & \text { if } q=3\end{cases} \\
& H^{q}\left(X, \mathbb{Z}_{X}(2)\right)= \begin{cases}0, & \text { if } q \leq 0 ; \\
K_{3}(F(X))_{\text {ind }}, & \text { if } q=1 ; \\
A^{0}\left(X, K_{2}\right), & \text { if } q=2 ; \\
A^{1}\left(X, K_{2}\right), & \text { if } q=3,\end{cases}
\end{aligned}
$$

where $K_{3}(L)_{\text {ind }}:=\operatorname{Coker}\left(K_{3}(L) \rightarrow K_{3}^{Q}(L)\right)$ for a field $L$.

Let $G$ be a semisimple algebraic group over $F$ and let $C$ be the kernel of a simply connected cover $G^{\text {sc }} \rightarrow G$.

Let $f: X \rightarrow Y$ be a $G$-torsor over a smooth geometrically irreducible variety $Y$ over $F$. We write $\mathbb{Z}_{f}(i)$ for the cone of the natural morphism $\mathbb{Z}_{Y}(i) \rightarrow$ $R f_{*} \mathbb{Z}_{X}(i)$ in the derived category $D^{+} \mathrm{Sh}_{\text {ét }}(Y)$ of étale sheaves of abelian groups on $Y$. Thus, we have the exact triangles

$$
\mathbb{Z}_{Y}(i) \longrightarrow R f_{*}\left(\mathbb{Z}_{X}(i)\right) \longrightarrow \mathbb{Z}_{f}(i) \longrightarrow \mathbb{Z}_{Y}(i)[1]
$$

4a. The complex $\mathbb{Z}_{f}(1)$. We have $\mathbb{Z}(1)=\mathbb{G}_{m}[-1]$, hence $\mathcal{H}^{i}\left(\mathbb{Z}_{f}(1)\right)=0$ for $i \leq 0$ and there is an exact sequence

$$
0 \longrightarrow \mathbb{G}_{m, Y} \longrightarrow f_{*}\left(\mathbb{G}_{m, X}\right) \longrightarrow \mathcal{H}^{1}\left(\mathbb{Z}_{f}(1)\right) \longrightarrow 0 .
$$

Therefore, $\mathcal{H}^{1}\left(\mathbb{Z}_{f}(1)\right)$ is the étale sheaf associated with the presheaf

$$
U \mapsto F\left[f^{-1} U\right]^{\times} / F[U]^{\times},
$$

and hence, it follows from $(1.1)$ that $\mathcal{H}^{1}\left(\mathbb{Z}_{f}(1)\right)=\widehat{G}=0$. (We view $\widehat{G}$ as a sheaf on $Y$ via the pull-back with respect to the structure morphism $Y \rightarrow \operatorname{Spec} F$.) 
If $q \geq 2$, we have $\mathcal{H}^{q}\left(\mathbb{Z}_{f}(1)\right)=R^{q} f_{*}\left(\mathbb{Z}_{X}(1)\right)$. Again, by $(1.1), \mathcal{H}^{2}\left(\mathbb{Z}_{f}(1)\right) \simeq \widehat{C}$ as the étale sheaves on $Y$. Thus, we have

$$
\mathcal{H}^{q}\left(\mathbb{Z}_{f}(1)\right)= \begin{cases}0, & \text { if } q \leq 1 \\ \widehat{C}, & \text { if } q=2 .\end{cases}
$$

It follows that

$$
\tau_{\leq 2} \mathbb{Z}_{f}(1) \simeq \mathcal{H}^{2}\left(\mathbb{Z}_{f}(1)\right)[-2]=\widehat{C}[-2]
$$

where $\tau_{\leq i}$ is the truncation functor. The exact sequence of the étale cohomology groups for the exact triangle (4.3) (when $i=1$ ) gives then the exact sequence (1.1).

Denote by $\beta_{f}$ the composition

$$
\beta_{f}: \widehat{C} \simeq \tau_{\leq 2} \mathbb{Z}_{f}(1)[2] \longrightarrow \mathbb{Z}_{f}(1)[2] \longrightarrow \mathbb{Z}_{Y}(1)[3] .
$$

4 b. The complex $\mathbb{Z}_{f}(2)$. The complex $\mathbb{Z}(2)$ is supported in dimensions 1 and 2 , hence $\mathcal{H}^{q}\left(\mathbb{Z}_{f}(2)\right)=0$ if $q<0$. We have an exact sequence

$$
\begin{aligned}
0 \longrightarrow \mathcal{H}^{0}\left(\mathbb{Z}_{f}(2)\right) \longrightarrow \mathcal{H}^{1}\left(\mathbb{Z}_{Y}(2)\right) \stackrel{s}{\longrightarrow} R^{1} f_{*}\left(\mathbb{Z}_{X}(2)\right) \longrightarrow \\
\\
\mathcal{H}^{1}\left(\mathbb{Z}_{f}(2)\right) \longrightarrow \mathcal{H}^{2}\left(\mathbb{Z}_{Y}(2)\right) \longrightarrow R^{2} f_{*}\left(\mathbb{Z}_{X}(2)\right) \longrightarrow \mathcal{H}^{2}\left(\mathbb{Z}_{f}(2)\right) \longrightarrow 0 .
\end{aligned}
$$

It follows from $(4.2)$ that $\mathcal{H}^{1}\left(\mathbb{Z}_{Y}(2)\right)$ and $R^{1} f_{*}\left(\mathbb{Z}_{X}(2)\right)$ are the étale sheaves associated to the presheaves

$U \mapsto H^{1}(U, \mathbb{Z}(2))=K_{3} F(U)_{\text {ind }} \quad$ and $\quad U \mapsto H^{1}\left(f^{-1} U, \mathbb{Z}(2)\right)=K_{3} F\left(f^{-1} U\right)_{\text {ind }}$,

respectively. The variety of $G$ is unirational by [2, Theorem 18.2], hence the natural morphism $K_{3} F(U)_{\text {ind }} \rightarrow K_{3} F\left(f^{-1} U\right)_{\text {ind }}$ is an isomorphism if the $G$ torsor $f^{-1} U \rightarrow U$ is trivial by [4, Lemma 6.2]. As the $G$-torsor $f$ is trivial locally in the étale topology, the first morphism $s$ in the sequence (4.6) is an isomorphism.

The sheaves $\mathcal{H}^{2}\left(\mathbb{Z}_{Y}(2)\right)$ and $R^{2} f_{*}\left(\mathbb{Z}_{X}(2)\right)$ are associated to the presheaves $U \mapsto H^{2}(U, \mathbb{Z}(2))=A^{0}\left(U, K_{2}\right) \quad$ and $\quad U \mapsto H^{2}\left(f^{-1} U, \mathbb{Z}(2)\right)=A^{0}\left(f^{-1} U, K_{2}\right)$,

respectively. As the $G$-torsor $f$ is trivial locally in the étale topology, it follows from Proposition 3.4 that we have an exact sequence

$$
0 \longrightarrow \mathcal{H}^{2}\left(\mathbb{Z}_{Y}(2)\right) \longrightarrow R^{2} f_{*}\left(\mathbb{Z}_{X}(2)\right) \longrightarrow \widehat{C} * \mu \longrightarrow 0 .
$$

Therefore, by (4.6),

$$
\mathcal{H}^{q}\left(\mathbb{Z}_{f}(2)\right)= \begin{cases}0, & \text { if } q \leq 1 \\ \widehat{C} * \mu, & \text { if } q=2\end{cases}
$$

The sheaf $\mathcal{H}^{3}\left(\mathbb{Z}_{f}(2)\right)=R^{3} f_{*}\left(\mathbb{Z}_{X}(2)\right)$ is associated with the presheaf

$$
U \mapsto H^{3}\left(f^{-1} U, \mathbb{Z}(2)\right)=A^{1}\left(f^{-1} U, K_{2}\right) .
$$

Proposition 3.5 yields then a morphism of étale sheaves

$$
\mathcal{H}^{3}\left(\mathbb{Z}_{f}(2)\right) \longrightarrow D(G) \text {. }
$$


Consider the product map

$$
\mathrm{CH}^{1}\left(f^{-1} U\right) \otimes A^{0}\left(U, K_{1}\right) \longrightarrow A^{1}\left(f^{-1} U, K_{2}\right) .
$$

Assume that $f^{-1} U \rightarrow U$ is a trivial $G$-torsor. Tensoring the exact sequence (see $(1.1))$

$$
0 \longrightarrow \mathrm{CH}^{1}(U) \longrightarrow \mathrm{CH}^{1}\left(f^{-1} U\right) \longrightarrow \widehat{C} \longrightarrow 0
$$

with $A^{0}\left(U, K_{1}\right)$ and sheafifying, we get a morphism of sheaves

$$
\widehat{C} \otimes \mathbb{G}_{m} \longrightarrow \mathcal{H}^{3}\left(\mathbb{Z}_{f}(2)\right) .
$$

It follows from Proposition 3.5 that the sequence of sheaves

$$
0 \longrightarrow \widehat{C} \otimes \mathbb{G}_{m} \longrightarrow \mathcal{H}^{3}\left(\mathbb{Z}_{f}(2)\right) \longrightarrow D(G) \longrightarrow 0
$$

is exact.

In particular,we have a canonical morphism

$$
\tau_{f}: \tau_{\leq 3} \mathbb{Z}_{f}(2)[3] \longrightarrow \mathcal{H}^{3}\left(\mathbb{Z}_{f}(2)\right) \longrightarrow D(G) .
$$

4c. The complex $\widehat{C}(1)$ and the morphism $\theta_{f}$. Let $f: X \rightarrow Y$ be a $G$ torsor. Write $\widehat{C}(1)$ for the derived tensor product $\widehat{C} \stackrel{L}{\otimes} \mathbb{Z}_{Y}(1)$. We have

$$
\mathcal{H}^{q}(\widehat{C}(1))= \begin{cases}\widehat{C} \otimes \mathbb{G}_{m}, & \text { if } q=1 ; \\ \widehat{C} * \mu, & \text { if } q=0 ; \\ 0, & \text { otherwise. }\end{cases}
$$

Let $l$ be the characteristic exponent of $F$. Note that the $l$-primary component of $\widehat{C} * \mu$ is trivial and the $l^{\prime}$-primary component of $C \otimes \mathbb{G}_{m}$ is trivial for every prime $l^{\prime} \neq l$. Hence

$$
\operatorname{Hom}\left(\widehat{C} \otimes \mathbb{G}_{m},(\widehat{C} * \mu)[i]\right)=0
$$

for every $i$ and

$$
\widehat{C}(1)=(\widehat{C} * \mu) \oplus\left(\widehat{C} \otimes \mathbb{G}_{m}\right)[-1] .
$$

It follows from (4.7) and (4.9) that we have the following diagram of the horizontal and the vertical exact triangles

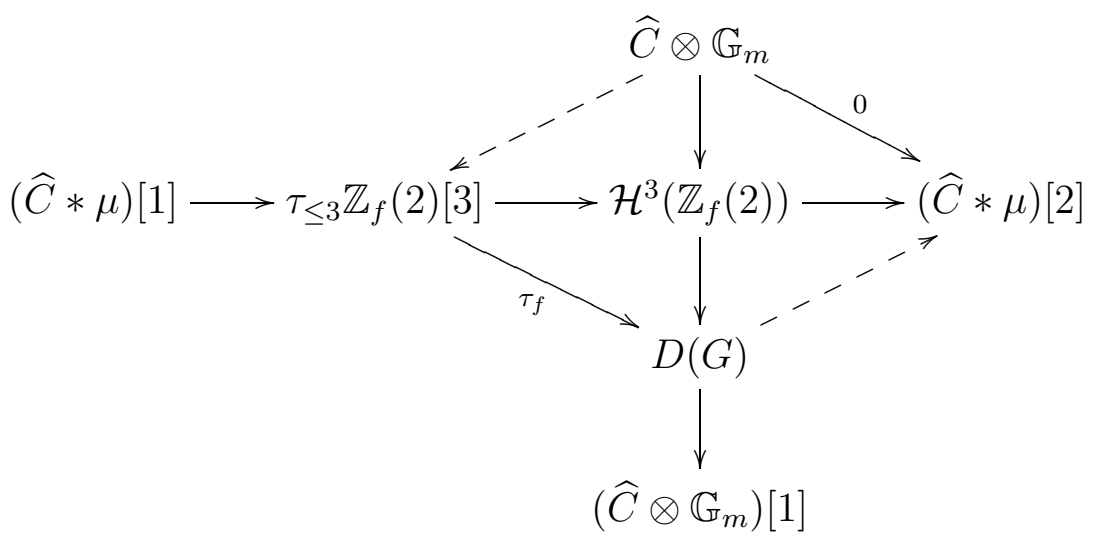


By (4.10), there are unique dotted arrows making the diagram commutative. It follows from the octahedral axiom that the diagram yields a natural exact triangle

$$
\widehat{C}(1)[1] \stackrel{\rho_{f}}{\longrightarrow} \tau_{\leq 3} \mathbb{Z}_{f}(2)[3] \stackrel{\tau_{f}}{\longrightarrow} D(G) \stackrel{\theta_{f}}{\longrightarrow} \widehat{C}(1)[2]
$$

with $\rho_{f}$ and $\theta_{f}$ defined by the morphisms in the diagram.

If $f: G \rightarrow \operatorname{Spec} F$ is the (trivial) $G$-torsor over $\operatorname{Spec} F$, we simply write $\theta_{G}$ for $\theta_{f}$. We view $\theta_{G}$ as a morphism in $D^{+} \operatorname{Sh}_{\text {ét }}(F)$ or in $D^{+} \operatorname{Sh}_{\text {ét }}(Y)$ for any variety $Y$.

The morphism $\theta_{G}$ may not be trivial. But in certain cases it is zero. (See also Remark 5.5.)

Proposition 4.1. (1) If $G$ is simply connected, then $\theta_{G}=0$.

(2) If $G$ is split, then the induced map $\theta_{G}^{*}: D(G) \rightarrow H^{2}(F, \widehat{C}(1))$ is trivial.

Proof. (1) follows simply because $\widehat{C}=0,(2)$ is proved in Lemma 6.13.

\section{MAIN THEOREMS}

Let $G$ be a semisimple group over $F$. Recall that we have a morphism $\beta_{f}$ : $\widehat{C} \rightarrow \mathbb{Z}_{Y}(1)[3]$ associated to a $G$-torsor $f: X \rightarrow Y$. Consider the compositions

$$
\lambda_{f}: D(G) \stackrel{\kappa}{\longrightarrow} \widehat{C} * \widehat{C} \longrightarrow(\widehat{C} \stackrel{L}{\otimes} \widehat{C})[-1] \stackrel{\left(\operatorname{Id} \stackrel{L}{\otimes} \beta_{f}\right)[-1]}{\longrightarrow} \widehat{C}(1)[2]
$$

and

$$
\sigma_{f}: \widehat{C}(1)=\widehat{C} \otimes \stackrel{L}{\otimes} \mathbb{Z}_{Y}(1) \stackrel{\beta_{f} \stackrel{L}{\otimes I d}}{\longrightarrow}\left(\mathbb{Z}_{Y}(1) \stackrel{L}{\otimes} \mathbb{Z}_{Y}(1)\right)[3] \stackrel{m[3]}{\longrightarrow} \mathbb{Z}_{Y}(2)[3]
$$

where $m$ is the product defined in [8] and [9].

The following theorem relates the étale motivic cohomology of weight 2 and degree at most 4 for the varieties $X$ and $Y$.

Theorem 5.1. Let $G$ be a semisimple group over a field $F, C$ the kernel of the universal cover $G^{\mathrm{sc}} \rightarrow G$ and $X \rightarrow Y$ a $G$-torsor with $Y$ a smooth geometrically irreducible variety. Then there are exact sequence:

$$
0 \longrightarrow A^{0}\left(Y, K_{2}\right) \longrightarrow A^{0}\left(X, K_{2}\right) \stackrel{\alpha}{\longrightarrow}(\widehat{C} * \mu)(F) \stackrel{\sigma_{f}^{*}}{\longrightarrow} A^{1}\left(Y, K_{2}\right) \longrightarrow A^{1}\left(X, K_{2}\right)
$$


and a commutative diagram

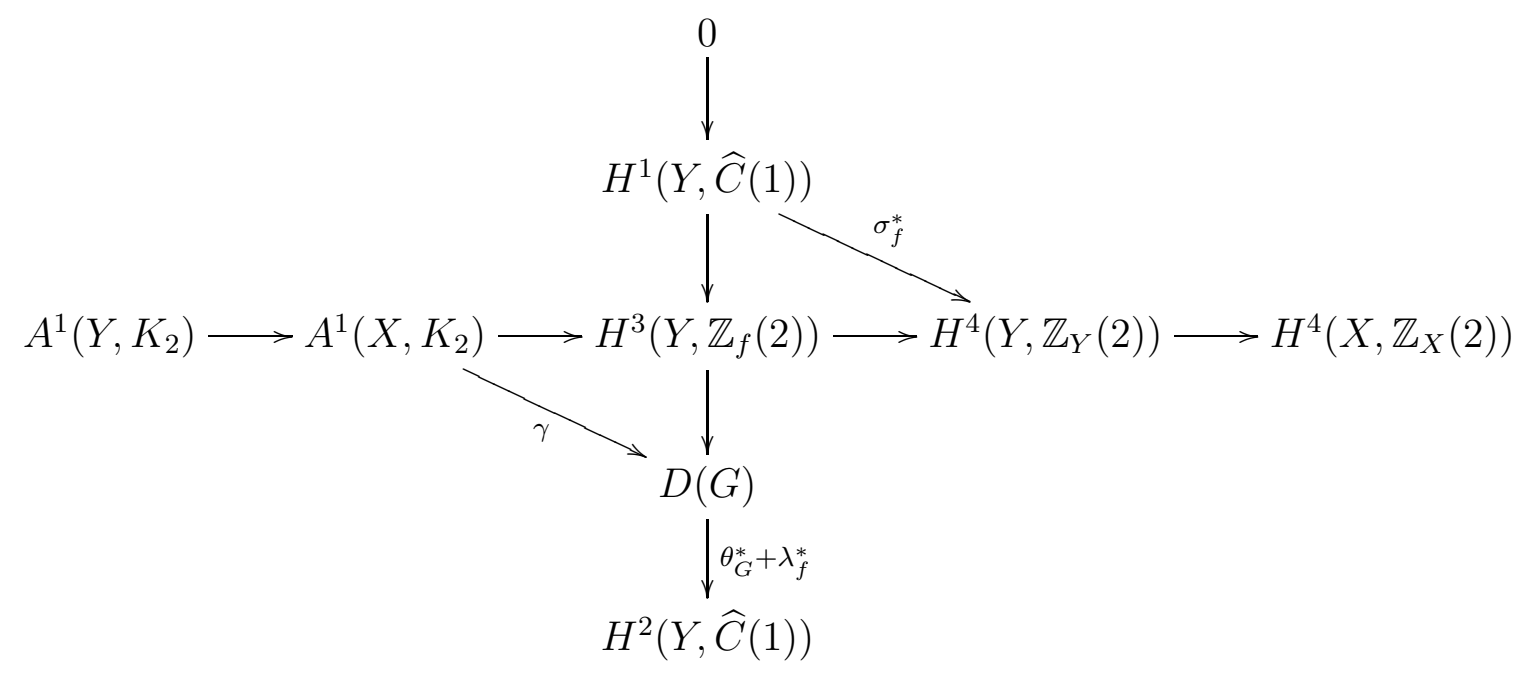

with the exact row and the column, where $\alpha$ and $\gamma$ are defined in (3.2) and (3.3), respectively.

Proof. It follows from (4.7) that

$$
H^{q}\left(Y, \mathbb{Z}_{f}(2)\right)= \begin{cases}0, & \text { if } q \leq 1 \\ \widehat{C} * \mu, & \text { if } q=2\end{cases}
$$

The first exact sequence and the horizontal exact sequence in the diagram are obtained then by Proposition 6.3(1) and by applying the étale cohomology groups of $Y$ to the exact triangle (4.3).

The vertical exact sequence is given by the exact triangle (4.12) since $H^{3}\left(Y, \tau_{\leq 3} \mathbb{Z}_{f}(2)\right)=H^{3}\left(Y, \mathbb{Z}_{f}(2)\right)$. The other marked homomorphisms in the diagram are identified in Sections $6 \mathrm{~d}$ and $6 \mathrm{e}$.

Remark 5.2. For a character $\chi \in \widehat{C}(F)$ and $a \in F[Y]^{\times}=H^{1}\left(Y, \mathbb{Z}_{Y}(1)\right)$, we have $\chi \cup(a) \in H^{1}(Y, \widehat{C}(1))$ and the map $\sigma_{f}^{*}: H^{1}(Y, \widehat{C}(1)) \longrightarrow H^{4}\left(Y, \mathbb{Z}_{Y}(2)\right)$ takes $\chi \cup(a)$ to $\beta_{f}^{*}(\chi) \cup(a)$, where

$$
\beta_{f}^{*}: \widehat{C}(F) \longrightarrow H^{3}\left(Y, \mathbb{Z}_{Y}(1)\right)=\operatorname{Br}(Y)
$$

is induced by $\beta_{f}$.

5a. Cohomology of classifying spaces. Let $G$ be a semisimple group over $F$. Choose a generically free representation $V$ of $G$ such that there is a $G$ equivariant open subset $U \subset V$ with the property $\operatorname{codim}_{V}(V \backslash U) \geq 3$ and a versal $G$-torsor $f: U \rightarrow Z$ (see [3, Lemma 9]). Moreover, we may assume that $Z(F) \neq \emptyset$.

By the assumption on codimension, it follows from the localization sequence that the restriction homomorphisms $A^{i}\left(V, K_{2}\right) \rightarrow A^{i}\left(U, K_{2}\right)$ are isomorphisms. Hence, by the homotopy invariance property,

$$
A^{i}\left(U, K_{2}\right)= \begin{cases}K_{2}(F), & \text { if } i=0 \\ 0, & \text { otherwise }\end{cases}
$$


By Theorem 5.1 applied to $f: U \rightarrow Z$, we have

$$
A^{i}\left(Z, K_{2}\right)= \begin{cases}K_{2}(F), & \text { if } i=0 \\ (\widehat{C} * \mu)(F), & \text { if } i=1 .\end{cases}
$$

We see that the groups $A^{i}\left(Z, K_{2}\right)$ are independent of the choice of $V$, and we write $A^{i}\left(B G, K_{2}\right)$ for $A^{i}\left(Z, K_{2}\right)$. We think of $Z$ as an approximation of the "classifying space" $B G$.

It follows from (4.2) and (5.4) that the groups $H^{q}(Z, \mathbb{Z}(2))$ don't depend on the choice of $V$ when $q=2$ or 3 . We write $H^{q}(B G, \mathbb{Z}(2))$ for these groups. But when $l=\operatorname{char}(F)>0$, the $l$-primary component of $H^{4}(Z, \mathbb{Z}(2))$, does depend on the choice of the representation $V$, so we have to modify the definition of $H^{4}(B G, \mathbb{Z}(2))$.

Let $A^{\bullet}$ be a cosimplicial abelian group and write $h_{*}\left(A^{\bullet}\right)$ for the homology groups of the associated complex of abelian groups. If $A^{\bullet}$ is a constant cosimplicial abelian group, we have $h_{0}\left(A^{\bullet}\right)=A^{0}$ and $h_{i}\left(A^{\bullet}\right)=0$ for all $i>0$.

Write $U^{n}$ for the product of $n$ copies of $U$ with the diagonal action of $G$. Let $H: \operatorname{SmVar}(F) \longrightarrow \mathrm{Ab}$ be a contravariant functor from the category of smooth varieties over $F$ to the category of abelian groups. We have the two maps $H\left(p_{i}\right): H(Z) \longrightarrow H\left(U^{2} / G\right), i=1,2$, where $p_{i}: U^{2} / G \rightarrow Z$ are the two projections. An element $h \in H(Z)$ is called balanced if $H\left(p_{1}\right)(h)=H\left(p_{2}\right)(h)$. We write $H(Z)_{\text {bal }}$ for the subgroup of balanced elements in $H(Z)$. In other words, $H(Z)_{\text {bal }}=h_{0}\left(H\left(U^{\bullet} / G\right)\right)$ (see [1]).

By Theorem 5.1, applied to the $G$-torsor $U^{n} \rightarrow U^{n} / G$ for every $n$, we have an exact sequence of cosimplicial groups

$0 \longrightarrow H^{1}\left(U^{\bullet} / G, \widehat{C}(1)\right) \longrightarrow H^{3}\left(U^{\bullet} / G, \mathbb{Z}_{f} \bullet(2)\right) \longrightarrow D(G) \longrightarrow H^{2}\left(U^{\bullet} / G, \widehat{C}(1)\right)$,

where the third group is viewed as a constant cosimplicial group. By Proposition $6.3(2), H^{1}\left(U^{n} / G, \widehat{C}(1)\right) \simeq H^{1}(F, \widehat{C}(1))$ for every $n$, hence the first cosimplicial group in the sequence is also constant. Applying $h_{0}$, we have an exact sequence (see [1, Lemma A.2])

$$
0 \longrightarrow H^{1}(F, \widehat{C}(1)) \longrightarrow H^{3}\left(Z, \mathbb{Z}_{f}(2)\right)_{\text {bal }} \longrightarrow D(G) \stackrel{\theta_{G}^{*}+\lambda_{f}^{*}}{\longrightarrow} H^{2}(Z, \widehat{C}(1)) .
$$

It follows from Theorem 5.1 applied to $f$ that we have an exact sequence

$$
0 \longrightarrow H^{1}(F, \widehat{C}(1)) \longrightarrow H^{3}\left(Z, \mathbb{Z}_{f}(2)\right) \longrightarrow D(G) \stackrel{\theta_{G}^{*}+\lambda_{f}^{*}}{\longrightarrow} H^{2}(Z, \widehat{C}(1)) .
$$

Therefore,

$$
H^{3}\left(Z, \mathbb{Z}_{f}(2)\right)_{\text {bal }}=H^{3}\left(Z, \mathbb{Z}_{f} \bullet(2)\right),
$$

i.e., every element in $H^{3}\left(Z, \mathbb{Z}_{f}(2)\right)$ is balanced.

By Theorem 5.1 again and (5.3), the sequence

$$
0 \longrightarrow H^{3}\left(U^{\bullet} / G, \mathbb{Z}_{f} \bullet(2)\right) \longrightarrow H^{4}\left(U^{\bullet} / G, \mathbb{Z}(2)\right) \longrightarrow H^{4}\left(U^{\bullet}, \mathbb{Z}(2)\right)
$$

is exact. Applying $h_{0}$ and taking into account (5.6), we get an exact sequence

$$
0 \longrightarrow H^{3}\left(Z, \mathbb{Z}_{f}(2)\right) \longrightarrow H^{4}(Z, \mathbb{Z}(2))_{\text {bal }} \longrightarrow H^{4}(U, \mathbb{Z}(2))_{\text {bal }} \text {. }
$$


By Lemma 6.8, the last group in the sequence is canonically isomorphic to $H^{4}(F, \mathbb{Z}(2))$. Write $\bar{H}^{4}(Z, \mathbb{Z}(2))$ for the factor group $\bar{H}^{4}(Z, \mathbb{Z}(2)) / H^{4}(F, \mathbb{Z}(2))$. It follows from (5.7) that there is an isomorphism

$$
H^{3}\left(Z, \mathbb{Z}_{f}(2)\right) \stackrel{\sim}{\rightarrow} \bar{H}^{4}(Z, \mathbb{Z}(2))_{\text {bal }}
$$

It follows from the proof of the following theorem that the group $H^{4}(Z, \mathbb{Z}(2))_{\text {bal }}$ does not depend on the choice of the representation $V$. We will denote this group by $H^{4}(B G, \mathbb{Z}(2))$. We write $B G$ for $Z$ and $E G$ for $U$.

Theorem 5.3. Let $G$ be a semisimple group over $F$ and $C$ the kernel of the universal cover $G^{\mathrm{sc}} \rightarrow G$. Then there are canonical isomorphisms

$$
H^{q}(B G, \mathbb{Z}(2)) \simeq A^{q-2}\left(B G, K_{2}\right) \simeq \begin{cases}K_{2}(F), & \text { if } q=2 ; \\ (\widehat{C} * \mu)(F), & \text { if } q=3,\end{cases}
$$

and an exact sequence

$$
0 \longrightarrow H^{1}(F, \widehat{C}(1)) \longrightarrow \bar{H}^{4}(B G, \mathbb{Z}(2)) \longrightarrow Q(G) \stackrel{\theta_{G}^{*}}{\longrightarrow} H^{2}(F, \widehat{C}(1)) .
$$

Proof. The first part follows from (4.2) and (5.4). By (5.5) and (5.8) it suffices to show that the kernel of $\theta_{G}^{*}+\lambda_{f}^{*}$ coincides with the kernel of $\theta_{G}^{*}: Q(G) \rightarrow$ $H^{2}(F, \widehat{C}(1))$. Let $a \in D(G)$ be such that $\theta_{G}^{*}(a)+\lambda_{f}^{*}(a)=0$ in $H^{2}(Z, \widehat{C}(1))$. Passing to $Z_{\text {sep }}$, we get $\lambda_{f}^{*}(a)=0$ in $H^{2}\left(Z_{\text {sep }}, \widehat{C}(1)\right)$. It follows from Lemma 6.6 that $a \in Q(G)=\operatorname{Ker}(D(G) \stackrel{\kappa}{\longrightarrow}(\widehat{C} * \widehat{C})(F))$ and $a \in \operatorname{Ker}\left(\theta_{G}^{*}\right)$ by Proposition $6.3(2)$.

Remark 5.4. If $G$ is simply connected, i.e., $\widehat{C}=0$, we get an isomorphism $\bar{H}^{4}(B G, \mathbb{Z}(2)) \simeq Q(G)$. This was proved in [4, Theorem B.5].

Remark 5.5. Let $X \rightarrow \operatorname{Spec} F$ be a $G$-torsor and $G^{\prime}=\operatorname{Aut}_{G}(X)$. As $X$ is trivial over $F_{\text {sep }}$, the groups $G$ and $G^{\prime}$ are inner twisted forms of each other, i.e., the group $G^{\prime}$ is the twist of $G$ by the torsor $X$. Then $E G^{\prime}$ and $B G^{\prime}$ are the twists of $E G$ and $B G$ by $X$, respectively. But this twist does not affect $B G$, i.e., $B G=B G^{\prime}$. It follows that $\operatorname{Ker}\left(\theta_{G}^{*}\right)=\operatorname{Ker}\left(\theta_{G^{\prime}}^{*}\right)$ since this equality holds over $F_{\text {sep }}$ and $Q(G)=Q\left(F_{\text {sep }}\right)^{\Gamma_{F}}$. In particular, if $\theta_{G}^{*}=0$, then $\theta_{G^{\prime}}^{*}=0$. As every adjoint semisimple group of inner type is an inner twisted form of a split group, it follows from Proposition 4.1 that $\theta_{G}^{*}=0$ for such a group $G$.

\section{ApPEndix}

6a. Homotopy invariant functors. A contravariant functor $S: \operatorname{SmVar}(F) \rightarrow$ $\mathrm{Ab}$ is called homotopy invariant if for every variety $X$, the map $S(X) \rightarrow$ $S\left(X \times \mathbb{A}_{F}^{1}\right)$ induced by the projection $X \times \mathbb{A}_{F}^{1} \rightarrow X$, is an isomorphism.

Example 6.1. The functors $X \mapsto A^{i}\left(X, K_{j}\right)$ are homotopy invariant by $[13$, $\S 9]$. It follows that the functor

$$
X \mapsto \operatorname{Coker}\left(\mathrm{CH}^{1}(X) \otimes F^{\times} \longrightarrow A^{1}\left(X, K_{2}\right)\right) .
$$

is also homotopy invariant. 
Lemma 6.2. Let $S: \operatorname{SmVar}(F) \rightarrow \mathrm{Ab}$ be a homotopy invariant contravariant functor and $G$ a semisimple group acting on a variety $X$ over $F$. Assume that map $S(X) \rightarrow S\left(X_{L}\right)$ is injective for every field extension $L / F$. Then the natural action of the group $G(F)$ on $S(X)$ is trivial.

Proof. By assumption, we can replace $F$ by an algebraic closure of $F$ and $G$ by a semisimple cover $G^{\text {sc }}$ of $G$ since the homomorphism $G^{\mathrm{sc}}(F) \rightarrow G(F)$ is surjective if $F$ is algebraically closed. As $G$ is simply connected, $G(F)$ is generated by the $F$-points of 1-dimensional unipotent subgroups $U \simeq \mathbb{A}_{F}^{1}$ of $G$. It suffices to show that every $t \in U(F)$ acts trivially on $S(X)$. The action morphism $a: U \times X \rightarrow X$ and the projection $p: U \times X \rightarrow X$ induce the maps $S(a), S(p): S(X) \rightarrow S(U \times X)$. By homotopy invariance, $S(p)$ is an isomorphism.

Write $i_{t}: X \rightarrow U \times X$ for the morphism $x \mapsto(t, x)$. As $a \circ i_{1}=p \circ i_{1}=\operatorname{Id}_{X}$, we have $S(a) \circ S\left(i_{1}\right)=\operatorname{Id}_{S(X)}=S(p) \circ S\left(i_{1}\right)$ and hence $S(a)=S(p)$ since $S(p)$ is an isomorphism. It follows that

$$
S\left(a \circ i_{t}\right)=S\left(i_{t}\right) \circ S(a)=S\left(i_{t}\right) \circ S(p)=S\left(p \circ i_{t}\right)=S\left(\operatorname{Id}_{X}\right)=\operatorname{Id}_{S(X)},
$$

whence the result as $t$ acts on $S(X)$ via $S\left(a \circ i_{t}\right)$.

6b. Cohomology of $\widehat{C}(1)$. Assume first that the group $G$ is split. Let $T$ be a split maximal torus in $G$. The exact triangle

$$
\widehat{T}(1) \longrightarrow \widehat{T}^{\mathrm{sc}}(1) \longrightarrow \widehat{C}(1) \longrightarrow \widehat{T}(1)[1]
$$

and (4.1) yield the triviality of $H^{i}(Y, \widehat{C}(1))$ for every variety $Y$ if $i<0$, an isomorphism

$$
H^{0}(Y, \widehat{C}(1)) \simeq \widehat{C} * A^{0}\left(Y, K_{1}\right)=\widehat{C} * \mu(F(Y))
$$

and the exact sequences

$$
\begin{aligned}
& 0 \longrightarrow \widehat{C} \otimes A^{0}\left(Y, K_{1}\right) \longrightarrow H^{1}(Y, \widehat{C}(1)) \longrightarrow \widehat{C} * \mathrm{CH}^{1}(Y) \longrightarrow 0, \\
& 0 \longrightarrow \widehat{C} \otimes \mathrm{CH}^{1}(Y) \longrightarrow H^{2}(Y, \widehat{C}(1)) \longrightarrow \widehat{C} * \mathrm{Br}(Y) \longrightarrow 0 .
\end{aligned}
$$

Now consider the general case when $G$ is not necessarily split. We have a natural homomorphism

$$
H^{2}(Y, \widehat{C}(1)) \longrightarrow H^{2}\left(Y_{\text {sep }}, \widehat{C}(1)\right)^{\Gamma_{F}} \longrightarrow\left(\widehat{C}_{\text {sep }} * \operatorname{Br}\left(Y_{\text {sep }}\right)\right)^{\Gamma_{F}} .
$$

Proposition 6.3. Let $Y$ be an absolutely irreducible variety over $F$. Then

(1) $H^{0}(Y, \widehat{C}(1)) \simeq(\widehat{C} * \mu)(F)$.

(2) Assume in addition that $A^{0}\left(Y_{\text {sep }}, K_{1}\right)=F_{\text {sep }}^{\times}, \mathrm{CH}^{1}\left(Y_{\text {sep }}\right)=0$ and $Y$ has a smooth F-point. Then the natural homomorphism $H^{1}(F, \widehat{C}(1)) \rightarrow$ $H^{1}(Y, \widehat{C}(1))$ is an isomorphism and the sequence

$0 \longrightarrow H^{2}(F, \widehat{C}(1)) \longrightarrow H^{2}(Y, \widehat{C}(1)) \longrightarrow\left(C_{\text {sep }} * \operatorname{Br}\left(Y_{\text {sep }}\right)\right)^{\Gamma_{F}} \longrightarrow 0$

is exact. 
Proof. (1) The Hochschild-Serre spectral sequence

$$
E_{2}^{p, q}=H^{p}\left(F, H^{q}\left(Y_{\mathrm{sep}}, \widehat{C}(1)\right) \Longrightarrow H^{p+q}(Y, \widehat{C}(1))\right.
$$

and (6.1) over $F_{\text {sep }}$ give the isomorphism (1).

(2) The spectral sequence and (6.2) over $F_{\text {sep }}$ provide an exact sequence

$$
\begin{aligned}
0 \longrightarrow H^{1}(F, \widehat{C} * \mu) \longrightarrow H^{1}(Y, \widehat{C}(1)) \longrightarrow\left(\widehat{C} \otimes F_{\text {sep }}^{\times}\right)^{\Gamma_{F}} \longrightarrow & \\
H^{2}(F, \widehat{C} * \mu) & \longrightarrow H^{2}(Y, \widehat{C}(1)) .
\end{aligned}
$$

The last homomorphism in the sequence is injective as $Y$ has an $F$-point. It follows from (4.11) that the map $H^{1}(F, \widehat{C}(1)) \rightarrow H^{1}(Y, \widehat{C}(1))$ is an isomorphism.

In order to prove the last statement, notice that all the differentials in the spectral sequence coming to the line $q=0$ are trivial as $Y$ has a rational point. By (6.2), we have

$$
E_{2}^{2,1}=H^{2}\left(F, \widehat{C} \otimes F_{\text {sep }}^{\times}\right) .
$$

This group is trivial as $\widehat{C} \otimes F_{\text {sep }}^{\times}$is $l$-primary torsion, where $l$ is the characteristic exponent of $F$, and the cohomological $l$-dimension of $F$ is at most 1 if $l>0$. It follows that the differential $E_{2}^{0,2} \rightarrow E_{2}^{2,1}$ is trivial. Hence $H^{2}(Y, \widehat{C}(1))$ has a filtration with the factors $H^{2}(F, \widehat{C} * \mu), H^{1}\left(F, \widehat{C} \otimes F_{\text {sep }}^{\times}\right)$and $H^{0}\left(F, \widehat{C}_{\text {sep }} *\right.$ $\left.\operatorname{Br}\left(Y_{\text {sep }}\right)\right)$. The result follows from (4.11).

Corollary 6.4. In the assumptions of the proposition assume in addition that $Y$ is smooth. Then the natural homomorphism $H^{2}(Y, \widehat{C}(1)) \rightarrow H^{2}(F(Y), \widehat{C}(1))$ is injective.

Proof. It follows from (6.3) that $H^{2}\left(F_{\text {sep }}(Y), \widehat{C}(1)\right)=\widehat{C} * \operatorname{Br}\left(F_{\text {sep }}(Y)\right.$. Hence we have a commutative diagram

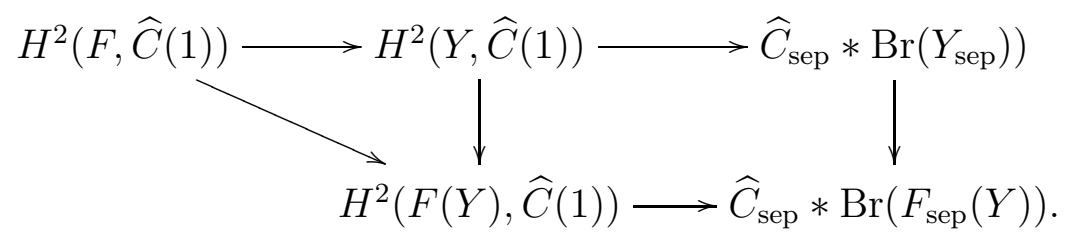

The top row is exact by Proposition 6.3 and the right vertical arrow is injective as $Y$ is smooth by [11, Corollary 2.6]. By specialization at a rational point, the diagonal map is injective. The result follows.

Lemma 6.5. Let $Y^{\prime} \rightarrow Y$ be a morphism of smooth varieties such that the induced maps $F_{\mathrm{sep}}[Y]^{\times} \rightarrow F_{\mathrm{sep}}\left[Y^{\prime}\right]^{\times}, \mathrm{CH}^{1}\left(Y_{\mathrm{sep}}\right) \rightarrow \mathrm{CH}^{1}\left(Y_{\mathrm{sep}}^{\prime}\right)$ are isomorphisms and the map $\operatorname{Br}\left(Y_{\text {sep }}\right) \rightarrow \operatorname{Br}\left(Y_{\text {sep }}^{\prime}\right)$ is injective. Then the map $H^{2}(Y, \widehat{C}(1)) \rightarrow$ $H^{2}\left(Y^{\prime}, \widehat{C}(1)\right)$ is injective.

Proof. Consider the natural morphism of the Hochschild-Serre spectral sequences for $Y$ and $Y^{\prime}$ and the complex $\widehat{C}(1)$. By assumption, the maps $E_{*}^{p, q}(Y) \rightarrow E_{*}^{p, q}\left(Y^{\prime}\right)$ are isomorphisms if $(p, q)=(1,1)$ and $(2,0)$ and injective if $(p, q)=(0,2)$. The result follows. 
Lemma 6.6. Let $f: X \rightarrow Y$ be a G-torsor over $F=F_{\text {sep }}$ with $Y$ smooth geometrically irreducible. If $\mathrm{CH}^{1}(X)=0$, then the composition

$$
(\widehat{C} * \widehat{C})(F) \longrightarrow H^{-1}(Y, \widehat{C} \stackrel{L}{\otimes} \widehat{C}) \stackrel{\cup \beta_{f}^{*}}{\longrightarrow} H^{2}(Y, \widehat{C}(1))
$$

is injective.

Proof. The exact sequence

$$
H^{2}(X, \mathbb{Z}(1)) \longrightarrow H^{2}\left(Y, \mathbb{Z}_{f}(1)\right) \longrightarrow H^{3}(Y, \mathbb{Z}(1))
$$

coincides with

$$
\mathrm{CH}^{1}(X) \longrightarrow \widehat{C} \stackrel{\beta_{f}^{*}}{\longrightarrow} \operatorname{Br}(Y)
$$

therefore, $\beta_{f}^{*}$ is injective.

The diagram

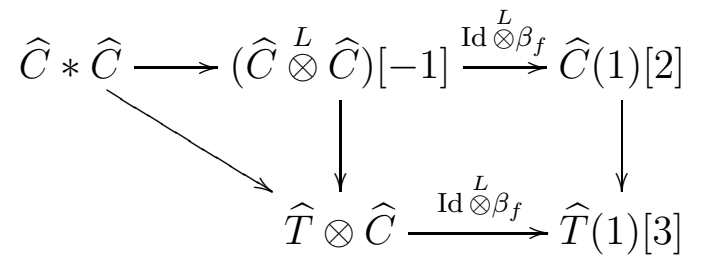

with the commutative triangle and anti-commutative square, where $T$ is a split maximal torus of $G$, yields the anti-commutative diagram

$$
\begin{aligned}
& \widehat{C} * \widehat{C} \longrightarrow H^{2}(Y, \widehat{C}(1)) \\
& \widehat{T} \otimes \widehat{C} \stackrel{\cup \beta_{f}^{*}}{\longrightarrow} H^{3}(Y, \widehat{T}(1))=\widehat{T} \otimes \operatorname{Br}(Y) .
\end{aligned}
$$

It follows that the top arrow in the last diagram is injective.

6c. Balanced elements. Let $V$ be a representation of $G$ as in Section 5a. We consider the étale cohomology with coefficients in the complex $\mathbb{Q} / \mathbb{Z}(2)$ defined in $[6]$. Write $H^{i}(F(V), \mathbb{Q} / \mathbb{Z}(j))_{\text {bal }}$ for the subgroup of elements in $H^{i}(F(V), \mathbb{Q} / \mathbb{Z}(j))$ whose images in $H^{i}(F(V \times V), \mathbb{Q} / \mathbb{Z}(j))$ under the two natural embeddings of $F(V)$ into $F(V \times V)$ coincide.

Lemma 6.7. Let $V$ be a finite dimensional vector space over $F$. Then the natural map $H^{i}(F, \mathbb{Q} / \mathbb{Z}(j)) \rightarrow H^{i}(F(V), \mathbb{Q} / \mathbb{Z}(j))_{\text {bal }}$ is an isomorphism for all $i$ and $j$.

Proof. We prove by induction on $n=\operatorname{dim}(V)$. Write $F(V)$ as $F\left(\mathbb{P}^{1} \times \mathbb{A}^{n-1}\right)$. By [1, Proposition A.9], every element $a \in H^{i}(F(V), \mathbb{Q} / \mathbb{Z}(j))_{\text {bal }}$ is unramified with respect to every point in $\mathbb{P}^{1} \times \mathbb{A}^{n-1}$ of codimension 1 . Therefore, $a$ is unramified with respect to all discrete valuations of $L\left(\mathbb{P}^{1}\right)$ over $L=F\left(\mathbb{A}^{n-1}\right)$. By [1, Proposition 5.1], $a$ comes from an element $b \in H^{i}(L, \mathbb{Q} / \mathbb{Z}(j))$ which is balanced as $H^{i}$ is injective for purely transcendental extensions. By induction, $b$ comes from an element in $H^{i}(F, \mathbb{Q} / \mathbb{Z}(j))$. 
Let $U \subset V$ be an open subvariety as in Section 5a. Write $H^{4}(U, \mathbb{Z}(2))_{\text {bal }}$ for the subgroup of elements $a \in H^{4}(U, \mathbb{Z}(2))$ such that $q_{1}^{*}(a)=q_{2}^{*}(a)$, where $q_{i}: U \times U \rightarrow U$ are two projections.

Lemma 6.8. The natural map $H^{4}(F, \mathbb{Z}(2)) \rightarrow H^{4}(U, \mathbb{Z}(2))_{\text {bal }}$ is an isomorphism.

Proof. By [6, Theorem 1.1], there is an exact sequence

$$
0 \longrightarrow \mathrm{CH}^{2}(U) \longrightarrow H^{4}(U, \mathbb{Z}(2)) \longrightarrow H_{Z a r}^{0}\left(U, \mathcal{H}^{3}(\mathbb{Q} / \mathbb{Z}(2))\right)
$$

It follows from (5.3) that $\mathrm{CH}^{2}(U)=0$, so the last arrow in the sequence is an injection. Moreover, by [1, Proposition A.10], the natural homomorphism

$$
H_{\text {Zar }}^{0}\left(U, \mathcal{H}^{3}(\mathbb{Q} / \mathbb{Z}(2))\right) \longrightarrow H^{4}(F(U), \mathbb{Q} / \mathbb{Z}(2))=H^{4}(F(V), \mathbb{Q} / \mathbb{Z}(2))
$$

is also injective. Thus, $H^{4}(U, \mathbb{Z}(2))$ injects into $H^{4}(F(V), \mathbb{Q} / \mathbb{Z}(2))$. The balanced elements in $H^{4}(U, \mathbb{Z}(2))$ correspond to the balanced elements in $H^{4}(F(V), \mathbb{Q} / \mathbb{Z}(2))$ which are constant by Lemma 6.7 .

6d. The maps $H^{i}(Y, \widehat{C}(1)) \rightarrow H^{i+3}(Y, \mathbb{Z}(2))$. We will prove that the composition

$$
\widehat{C}(1) \stackrel{\rho_{f}}{\longrightarrow} \tau_{\leq 3} \mathbb{Z}_{f}(2)[2] \longrightarrow \mathbb{Z}_{f}(2)[2] \longrightarrow \mathbb{Z}_{Y}(2)[3]
$$

that defines the map $H^{1}(Y, \widehat{C}(1)) \rightarrow H^{4}(Y, \mathbb{Z}(2))$ is equal to the composition (5.2) after localizing at $l$ and $(l)$, where $l$ is the characteristic exponent of $F$.

Case 1: We localize at $l$ all sheaves and groups below. Choose a positive integer $n$ prime to $l$ such that $n \cdot \widehat{C}=0$ and let $\mu_{n, f}^{\otimes i}$ be the cone of the natural morphism $\mu_{n, Y}^{\otimes i} \rightarrow R f_{*}\left(\mu_{n, Y}^{\otimes i}\right)$. Write $\mu_{n, f}$ for $\mu_{n, f}^{\otimes 1}$.

Recall (see [8] and [9]) that for $i=1,2, \mu_{n}^{\otimes i}$ is the cone of the morphism $\mathbb{Z}(i) \stackrel{n}{\longrightarrow} \mathbb{Z}(i)$ of multiplication by $n$. It follows that $\mu_{n, f}^{\otimes i}$ is the cone of $\mathbb{Z}_{f}(i) \stackrel{n}{\longrightarrow} \mathbb{Z}_{f}(i)$. By $(4.4)$,

$$
\mathcal{H}^{q}\left(\mu_{n, f}\right)= \begin{cases}0, & \text { if } q \leq 0 \\ \widehat{C}, & \text { if } q=1\end{cases}
$$

Denote by $\beta^{\prime}: \widehat{C} \rightarrow \mu_{n, Y}[2]$ the composition in the top row of the diagram

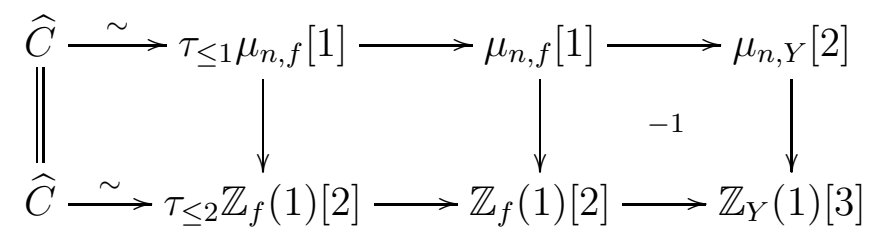

with the right square anti-commutative. It follows that the composition of $\beta^{\prime}$ with the morphism $\mu_{n, Y}[2] \rightarrow \mathbb{Z}_{Y}(1)[3]$ coincides with $-\beta_{f}$. 
Moreover, there is a diagram of horizontal exact triangles (with $\mu_{n}=\mu_{n, Y}$ )

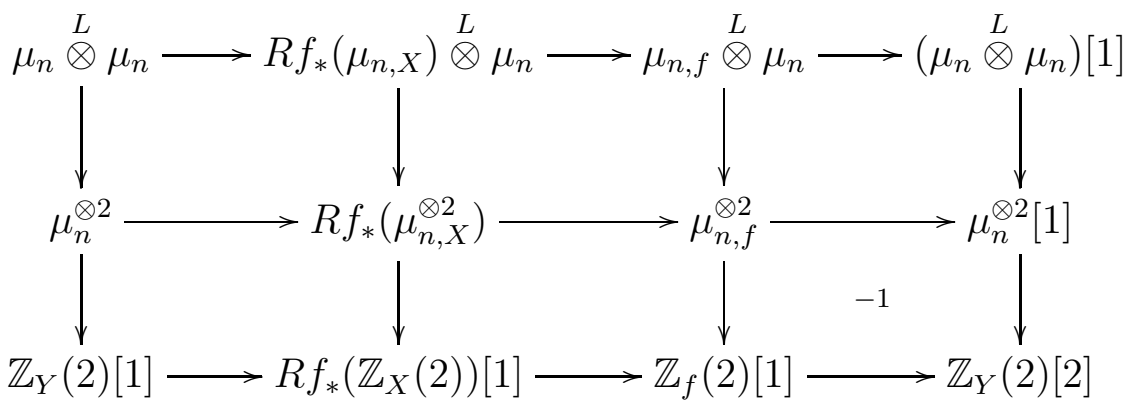

with the bottom right square anti-commutative and the other squares commutative.

Hence we have the diagram

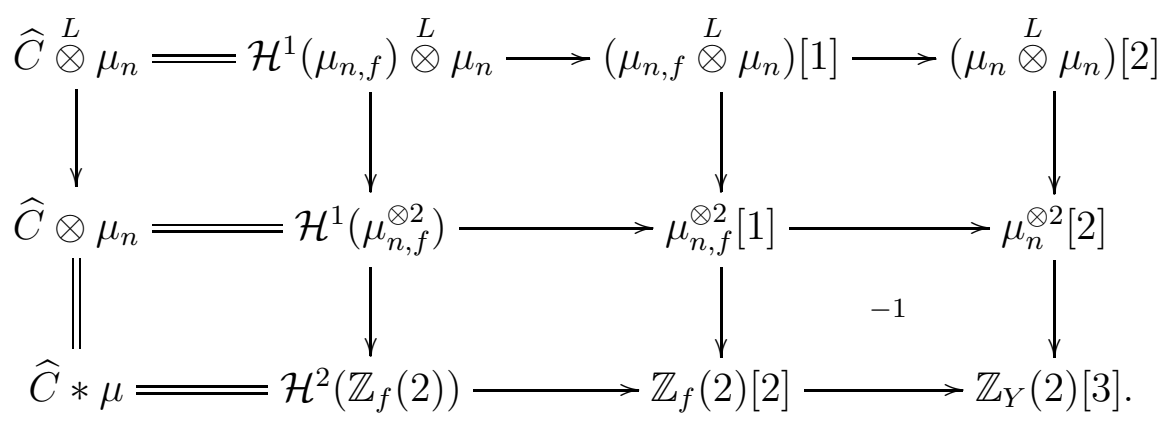

The composition of the morphism $\widehat{C}(1) \rightarrow \widehat{C} \stackrel{L}{\otimes} \mu_{n}$, the morphisms in the left column and the bottom row coincides with (6.4). The composition in the first row is equal to $\beta^{\prime} \otimes \mathrm{Id}$. It follows that (6.4) coincides with the negative of the composition

$$
\widehat{C}(1) \longrightarrow \widehat{C}^{\otimes} \mu_{n} \stackrel{\beta^{\prime} \otimes \mathrm{Id}}{\longrightarrow}\left(\mu_{n} \stackrel{L}{\otimes} \mu_{n}\right)[2] \longrightarrow \mathbb{Z}_{Y}(2)[3] .
$$

In the commutative diagram

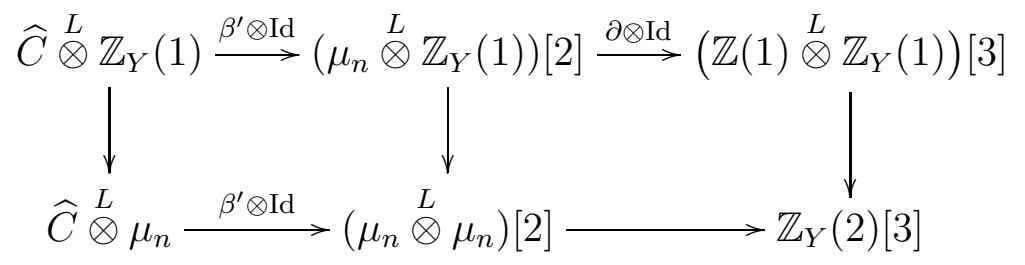

(see $\left[8\right.$, Remark 2.6]) the composition in the upper row coincides with $\left(-\beta_{f}\right) \otimes$ Id, hence the compositions (5.2) and (6.5) also coincide. 
Case 2: Now we localize at the ideal $(l)$. It follows immediately from the commutativity of the diagram

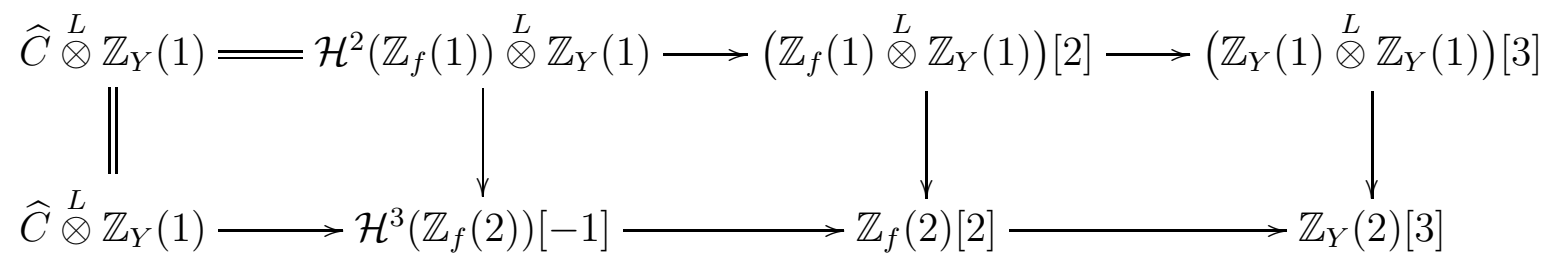

that the compositions (5.2) and (6.4) coincide.

6e. The map $D(G) \rightarrow H^{2}(Y, \widehat{C}(1))$. We prove that this map is equal to $\theta_{G}^{*}+\lambda_{f}^{*}$. For a geometrically irreducible variety $Y$ write $\widetilde{A}^{1}\left(G \times Y, K_{2}\right)$ for the cokernel of the product homomorphism

$$
\widehat{C} \otimes A^{0}\left(Y, K_{1}\right)=\mathrm{CH}^{1}(G) \otimes A^{0}\left(Y, K_{1}\right) \longrightarrow A^{1}\left(G \times Y, K_{2}\right) / A^{1}\left(Y, K_{2}\right) .
$$

By Proposition 3.5, if $G$ is split, there is a natural exact sequence

$$
0 \longrightarrow \widehat{C} * \mathrm{CH}^{1}(Y) \longrightarrow \widetilde{A}^{1}\left(G \times Y, K_{2}\right) \longrightarrow D(G) \longrightarrow 0 .
$$

This sequence is split canonically by the pull-back with respect to the projection $G \times Y \rightarrow G$, hence

$$
\widetilde{A}^{1}\left(G \times Y, K_{2}\right) \simeq\left(\widehat{C} * \mathrm{CH}^{1}(Y)\right) \oplus D(G) .
$$

Proposition 6.9. Let $G$ be a split semisimple group and let $X \rightarrow \operatorname{Spec} F$ be a trivial $G$-torsor with the action morphism $a: G \times X \rightarrow X$. Then the diagram

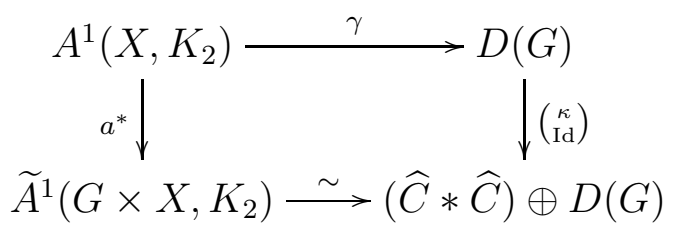

is commutative.

Proof. We may assume that $F=F_{\text {sep. }}$. The natural morphism $c: G_{F(X)} \rightarrow$ $G \times X$ yields the diagram

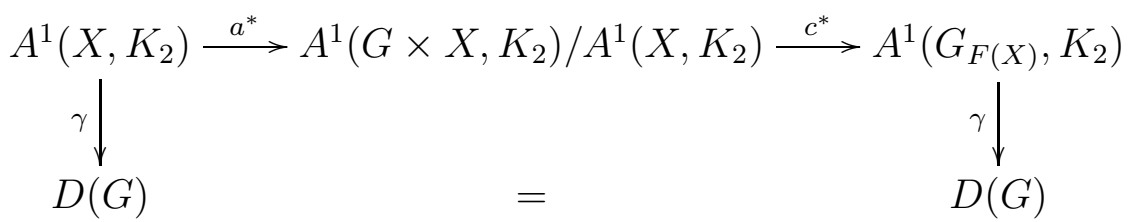

that is commutative by Proposition 3.3. It follows that the second components of both compositions in the diagram of the statement of the proposition are equal.

In order to prove that the first components of both compositions in the diagram are equal, we may assume that $X=G$. Let $T$ be a split maximal torus of $G$ and $Z:=G / T$. Considering spectral sequences for the two projections 
$G \times G \rightarrow G \times Z$ and $G \rightarrow Z$ as in Section 3a, we have the following commutative diagram

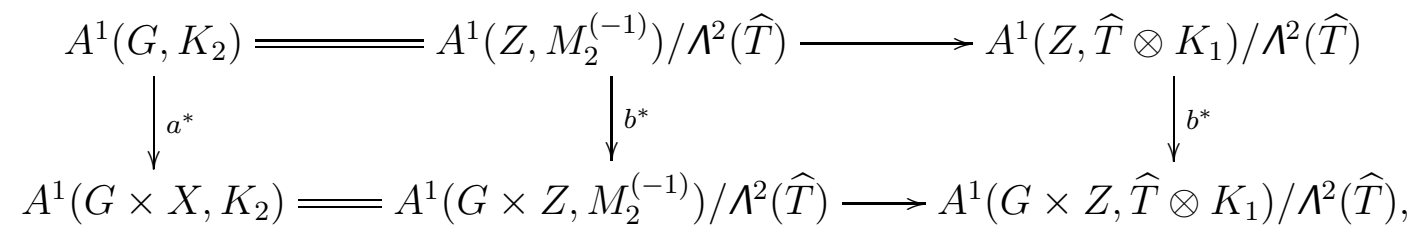

where $b: G \times Z \rightarrow Z$ is the action morphism. The homomorphism $b^{*}$ : $A^{1}\left(Z, \widehat{T} \otimes K_{1}\right) \rightarrow A^{1}\left(G \times Z, \widehat{T} \otimes K_{1}\right)$ coincides with $\widehat{T} \otimes \varphi$, where

$$
\varphi: \widehat{T}^{\mathrm{sc}}=\mathrm{CH}^{1}(Z) \longrightarrow \mathrm{CH}^{1}(G \times Z)=\mathrm{CH}^{1}(G) \oplus \mathrm{CH}^{1}(Z)=\widehat{C} \oplus \widehat{T}^{\mathrm{sc}},
$$

is given by the pull-back with respect to $b$. The first component $\varphi_{1}$ of $\varphi$ is the pull-back with respect to the morphism $\iota: G_{G(Z)} \rightarrow G \times Z \stackrel{b}{\longrightarrow} Z$ of the action on the generic point of $Z$. As the functor $\mathrm{CH}^{1}$ is homotopy invariant, by Lemma $6.2, \iota$ can be replace by the canonical morphism $G_{G(Z)} \rightarrow G \rightarrow Z$, hence $\varphi_{1}$ coincides with the canonical map $p: \widehat{T}^{\mathrm{sc}} \rightarrow \widehat{C}$. The commutativity of the diagram

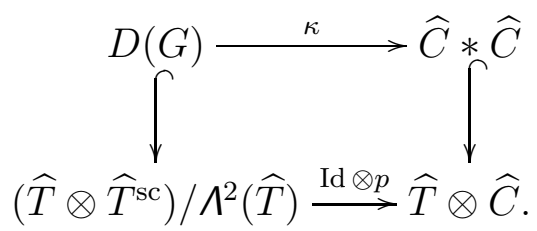

proves the statement.

Let $\left(C^{\bullet}, d^{\bullet}\right) \in D^{+} \mathrm{Sh}_{\text {ét }}(F)$. For an integer $n$, the exact sequence of $\Gamma_{F^{-}}$ modules

$$
0 \longrightarrow H^{n}\left(C^{\bullet}\right) \longrightarrow C^{n} / \operatorname{Im}\left(d_{n-1}\right) \stackrel{d^{n}}{\longrightarrow} \operatorname{Ker}\left(d^{n+1}\right) \longrightarrow H^{n+1}\left(C^{\bullet}\right) \longrightarrow 0
$$

yields a morphism

$$
\eta\left(C^{\bullet}\right): H^{n+1}\left(C^{\bullet}\right) \longrightarrow H^{n}\left(C^{\bullet}\right)[2]
$$

in $D^{+} \mathrm{Sh}_{\text {ét }}(F)$.

Let $X$ be a smooth variety over $F$ and $f: X \rightarrow$ Spec $F$ the structure morphism. By (4.1) and (4.2), we have for $i=1$ and 2 ,

$$
R^{q} f_{*}\left(\mathbb{Z}_{X}(i)\right)=H^{q}\left(X_{\mathrm{sep}}, \mathbb{Z}(i)\right)= \begin{cases}A^{0}\left(X_{\mathrm{sep}}, K_{i}\right), & \text { if } q=i \\ A^{1}\left(X_{\mathrm{sep}}, K_{i}\right), & \text { if } q=i+1 .\end{cases}
$$

Thus, applying the construction above to the complex $R f_{*}(\mathbb{Z}(i))$ and $n=1$ or 2 , we get the morphisms

$$
\eta_{X}^{(i)}:=\eta\left(R f_{*}(\mathbb{Z}(i))\right): A^{1}\left(X_{\mathrm{sep}}, K_{i}\right) \longrightarrow A^{0}\left(X_{\mathrm{sep}}, K_{i}\right)[2] .
$$

Lemma 6.10. Let $f: X \rightarrow \operatorname{Spec} F$ be a $G$-torsor. Then $A^{0}\left(X_{\mathrm{sep}}, K_{1}\right)=F_{\mathrm{sep}}^{\times}$, $A^{1}\left(X_{\mathrm{sep}}, K_{1}\right)=\widehat{C}$ and the morphism $\eta_{X}^{(1)}: \widehat{C} \rightarrow F_{\mathrm{sep}}^{\times}[2]=\mathbb{Z}(1)[3]$ coincides with $\beta_{f}$. 
Proof. Let $\mathbb{G}_{m, X} \rightarrow E^{\bullet}$ be an injective resolution. Then the complex $\mathbb{G}_{m, F} \rightarrow$ $f_{*}\left(E^{\bullet}\right)$ coincides with $\mathbb{Z}_{f}(1)$ (with $\mathbb{G}_{m}$ of degree zero). It follows that the first three terms of the exact sequence (6.6) for the complex $C^{\bullet}=R f_{*}\left(\mathbb{Z}_{X}(1)\right)$ and $n=1$ coincides with $\tau_{\leq 2} \mathbb{Z}_{f}(1)$. It follows that the morphism $\eta_{X}^{(1)}=\theta\left(C^{\bullet}\right)$ is equal to the composition $\widehat{C} \simeq \mathcal{H}^{2}\left(\mathbb{Z}_{f}(1)\right) \simeq \tau_{\leq 2} \mathbb{Z}_{f}(1)[2] \longrightarrow \mathbb{G}_{m, F}[2]=$ $\mathbb{Z}_{F}(1)[3]$, and hence is equal to $\beta_{f}$.

The Rost complex $C^{\bullet}\left(X, K_{i}\right)$ (see Section 1a) also yields a morphism

$$
\eta\left(C^{\bullet}\left(X, K_{i}\right)\right): A^{1}\left(X_{\mathrm{sep}}, K_{i}\right) \rightarrow A^{0}\left(X_{\mathrm{sep}}, K_{i}\right)[2] .
$$

Lemma 6.11. The morphisms $\eta_{X}^{(i)}$ and $\eta\left(C^{\bullet}\left(X, K_{i}\right)\right)$ coincide for $i=1$ and 2 .

Proof. Let $\mathcal{A}$ be the category of $\Gamma_{F}$-equivariant Zariski sheaves of abelian groups on $X_{\mathrm{sep}}$. The functor $R f_{*}$ is the composition of the functors

$$
D^{+} \operatorname{Sh}_{\text {ét }}(X) \stackrel{\alpha}{\longrightarrow} \mathcal{A} \stackrel{\delta}{\longrightarrow} D^{+} \operatorname{Sh}_{\text {ét }}(F),
$$

where $\alpha$ is the change of the site functor and $\delta$ is the functor of global sections. By [6, Lemma 1.4], $R^{i} \alpha(\mathbb{Z}(i))=K_{i}$, so that we have the following morphisms in $\mathcal{A}$ :

$$
R \alpha(\mathbb{Z}(i)) \longleftarrow \tau_{\leq i} R \alpha(\mathbb{Z}(i)) \longrightarrow K_{i} .
$$

Applying $R \delta$, we get the morphisms

$$
R f_{*}(\mathbb{Z}(i)) \longleftarrow R \delta\left(\tau_{\leq i} R \alpha(\mathbb{Z}(i))\right) \stackrel{\varphi}{\longrightarrow} R \delta\left(K_{i}\right) .
$$

Note that the left morphism induces isomorphisms on the homology in dimensions $\leq i$, hence the morphism $\eta$ for the complex $R \delta\left(\tau_{\leq i} R \alpha(\mathbb{Z}(i))\right)$ coincides with $\eta_{X}^{(i)}$. On the other hand, $R \delta\left(K_{i}\right)$ coincides with the Rost complex (Gersten's resolution, see $[12, \S 7])$, hence $\varphi$ yields the equality $\eta_{X}^{(i)}=$ $\theta\left(C^{\bullet}\left(X, K_{i}\right)\right)$.

Example 6.12. Let $f: X \rightarrow$ Spec $F$ be a $G$-torsor. The Rost complex for $i=1$ coincides with $F_{\text {sep }}(X) \rightarrow \operatorname{Div}\left(X_{\text {sep }}\right)$. It follows from Lemmas 6.10 and 6.11 that the morphism $\beta_{f}$ is induced by the exact sequence

$$
1 \longrightarrow F_{\text {sep }}^{\times} \longrightarrow F_{\text {sep }}(X)^{\times} \longrightarrow \operatorname{Div}\left(X_{\text {sep }}\right) \longrightarrow \widehat{C}_{\text {sep }} \longrightarrow 0 .
$$

Now consider the case $i=2$. We first prove the statement for torsors over Spec $F$.

Lemma 6.13. Let $f: X \rightarrow \operatorname{Spec} F$ be a $G$-torsor. Then

(1) $\theta_{f}=\theta_{G}+\lambda_{f}$.

(2) If $G$ is split, then $\theta_{G}$ induces the trivial homomorphism $D(G) \rightarrow H^{2}(F, \widehat{C}(1))$.

Proof. We drop the subscript "sep" in the proof. As before, write $C^{\bullet}\left(X, K_{*}\right)$ for the Rost complex of a variety $X$ and $\bar{C}\left(X, K_{*}\right)$ for the "reduced" complex

$$
\operatorname{Coker}\left(C^{\bullet}\left(\operatorname{Spec} F, K_{*}\right) \longrightarrow C^{\bullet}\left(X, K_{*}\right)\right)=C^{\bullet}\left(X, K_{*}\right) / K_{*}(F) \text {. }
$$


The reduced complex $\bar{C}^{\bullet}\left(G, K_{1}\right)$ coincides with $\operatorname{PDiv}(G) \hookrightarrow \operatorname{Div}(G)$ and hence is isomorphic to $\widehat{C}[-1]$. The complex $C^{\bullet}\left(X, K_{1}\right)$ is equal to $F(X)^{\times} \rightarrow$ $\operatorname{Div}(X)$ and hence by Lemmas 6.10 and 6.11 , fits into an exact triangle

$$
\mathbb{Z}_{X}(1)[1] \longrightarrow C^{\bullet}\left(X, K_{1}\right) \longrightarrow \widehat{C}[-1] \stackrel{\beta_{f}[-1]}{\longrightarrow} \mathbb{Z}_{X}(1)[2] .
$$

Write $C_{1}^{\bullet}$ for the tensor product $\bar{C}^{\bullet}\left(G, K_{1}\right) \otimes C^{\bullet}\left(X, K_{1}\right) \simeq \widehat{C}[-1] \otimes C^{\bullet}\left(X, K_{1}\right)$. Thus, we have the exact triangles

$$
\widehat{C}[-1] \stackrel{L}{\otimes} \mathbb{Z}_{X}(1)[1] \longrightarrow C_{1}^{\bullet} \longrightarrow \widehat{C}[-1] \stackrel{L}{\otimes} \widehat{C}[-1] \stackrel{\left(\mathrm{Id} \stackrel{L}{\otimes} \beta_{f}\right)[-1]}{\longrightarrow} \widehat{C}[-1] \stackrel{L}{\otimes} \mathbb{Z}_{X}(1)[2]
$$

and therefore

$$
\widehat{C}(1) \longrightarrow C_{1}^{\bullet} \longrightarrow\left(\widehat{C}^{\stackrel{L}{\otimes}} \widehat{C}\right)[-2] \stackrel{\left(\operatorname{Id} \stackrel{L}{\otimes} \beta_{f}\right)[-2]}{\longrightarrow} \widehat{C}(1)[1] .
$$

Note that $\tau_{\leq-1}(\widehat{C} \stackrel{L}{\otimes} \widehat{C})=(\widehat{C} * \widehat{C})[1]$. It follows that the triangle

$$
\widehat{C}(1) \longrightarrow \tau_{\leq 1}\left(C_{1}^{\bullet}\right) \longrightarrow(\widehat{C} * \widehat{C})[-1] \stackrel{\pi[-1]}{\longrightarrow} \widehat{C}(1)[1],
$$

where $\pi$ is the composition

$$
\pi: \widehat{C} * \widehat{C} \longrightarrow \widehat{(} C \stackrel{L}{\otimes} \widehat{C})[-1] \stackrel{\left(\operatorname{Id} \stackrel{L}{\otimes} \beta_{f}\right)[-1]}{\longrightarrow} C(1)[2],
$$

is exact. Note that $\pi \circ \kappa=\lambda_{f}$ by the definition of $\lambda_{f}$ in (5.1).

Write $C_{2}^{\bullet}$ for the complex

$$
\operatorname{Coker}\left(C^{\bullet}\left(X, K_{2}\right) \longrightarrow C^{\bullet}\left(G \times X, K_{2}\right)\right)
$$

induced by the projection $G \times X \rightarrow X$. The external product of cycles gives a morphism of complexes $\chi: C_{1}^{\bullet} \rightarrow C_{2}^{\bullet}$. The action morphism $a: G \times X \rightarrow X$ yields the composition of complexes

$$
\bar{C}^{\bullet}\left(X, K_{2}\right) \stackrel{a^{*}}{\longrightarrow} \bar{C}^{\bullet}\left(G \times X, K_{2}\right) \longrightarrow C_{2}^{\bullet}
$$

Similarly, the projection $p: G \times X \rightarrow G$ yields the morphism of complexes $p^{*}: \bar{C}^{\bullet}\left(G, K_{2}\right) \rightarrow C_{2}^{\bullet}$.

We have then the following diagram of morphisms of complexes

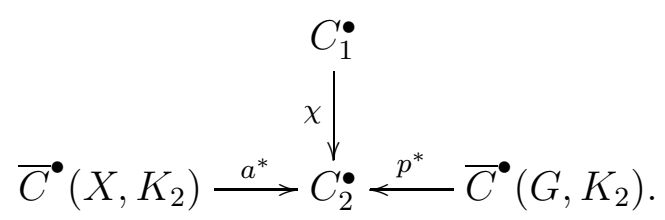

Note that all the morphisms in the diagram are isomorphisms on $H^{0}=\widehat{C} * \mu$.

We prove the proposition after localizing at $l$ or $(l)$, where $l$ is the characteristic exponent of $F$.

First localize at $l$. In particular, we have $\widehat{C} \otimes F^{\times}=0$ as $F$ is separably closed. 
By Proposition 3.5, we have $H^{1}\left(C_{2}^{\bullet}\right)=(\widehat{C} * \widehat{C}) \oplus D(G)$ and $H^{1}=D(G)$ for the other two complexes in the bottom row of the diagram (6.8). By Lemma 6.11, $\theta_{f}=\eta_{X}^{(2)}=\eta\left(\bar{C}^{\bullet}\left(X, K_{2}\right)\right)$ and $\theta_{G}=\eta_{G}^{(2)}=\eta\left(\bar{C}^{\bullet}\left(G, K_{2}\right)\right)$.

It follows from $(6.7)$ that $H^{1}\left(C_{1}^{\bullet}\right)=\widehat{C} * \widehat{C}$. Moreover, the morphism $\chi$ : $C_{1}^{\bullet} \rightarrow C_{2}^{\bullet}$ induces the identity on $H^{0}$ and the canonical inclusion on $H^{1}$. By (6.7), the morphism $\eta\left(C_{1}^{\bullet}\right): \widehat{C} * \widehat{C} \rightarrow \widehat{C}(1)[2]$ coincides with $\pi$. Hence the composition of the inclusion $\widehat{C} * \widehat{C} \hookrightarrow(\widehat{C} * \widehat{C}) \oplus D(G)$ and $\eta\left(C_{2}^{\bullet}\right)$ also coincides with $\pi$.

The map $p^{*}$ induces the canonical inclusion $D(G) \hookrightarrow(\widehat{C} * \widehat{C}) \oplus D(G)$ on $H^{1}$ and $a^{*}$ induces the map $\left(\begin{array}{c}\kappa \\ \text { Id }\end{array}\right): D(G) \hookrightarrow(\widehat{C} * \widehat{C}) \oplus D(G)$ by Proposition 6.9.

We have the following commutative diagram

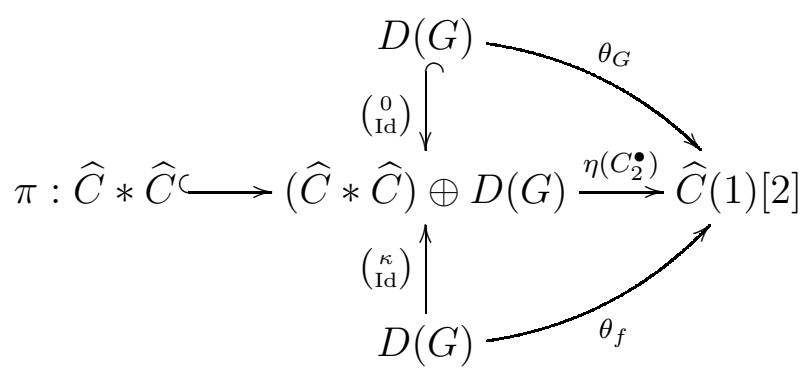

It follows that $\theta_{f}=\theta_{G}+\lambda_{f}$.

If $G$ is split, by Corollary 3.6, the rows of the diagram

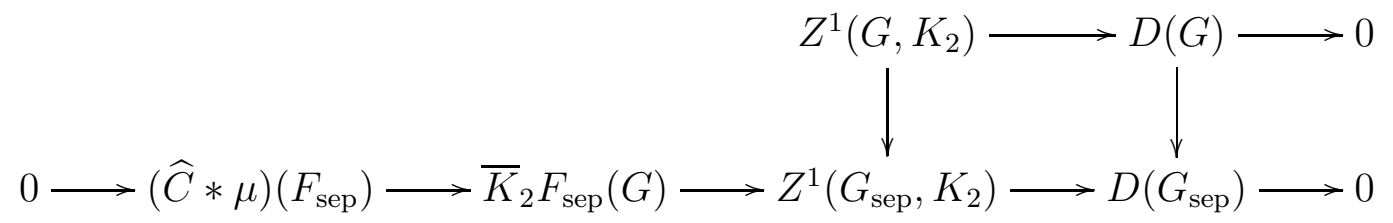

are exact. As $G$ is split, the right vertical homomorphism is an isomorphism. It follows that the map $Z^{1}\left(G_{\text {sep }}, K_{2}\right)^{\Gamma_{F}} \rightarrow D\left(G_{\text {sep }}\right)^{\Gamma_{F}}=D(G)$ is surjective. Hence the connecting homomorphism for the exact sequence in the diagram

$$
D(G)=D\left(G_{\mathrm{sep}}\right)^{\Gamma_{F}} \longrightarrow H^{2}(F, \widehat{C} * \mu)=H^{2}(F, \widehat{C}(1))
$$

is trivial.

Now localize at the ideal $(l)$. We have $\widehat{C} * \mu=0$ and $\widehat{C}(1)=\left(\widehat{C} \otimes F^{\times}\right)[-1]$. It follows from (6.7) that there is an exact sequence

$$
0 \longrightarrow \widehat{C} \otimes F^{\times} \longrightarrow H^{1}\left(C_{1}^{\bullet}\right) \longrightarrow \widehat{C} * \widehat{C} \longrightarrow 0 .
$$

By Proposition 3.5, the $H^{1}$-cohomology of all the complexes in the diagram (6.8) contain $\widehat{C} \otimes F^{\times}$and all the morphisms in the diagram induce the identity morphism on $\widehat{C} \otimes F^{\times}$. As above, the diagram (6.8) yields the diagram (6.9) with the morphism $\eta\left(C_{2}^{\bullet}\right)$ replaced by the one induced by the exact sequence

$$
0 \longrightarrow \widehat{C} \otimes F^{\times} \longrightarrow H^{1}\left(C_{2}^{\bullet}\right) \longrightarrow(\widehat{C} * \widehat{C}) \oplus D(G) \longrightarrow 0 .
$$

Therefore, again $\theta_{f}=\theta_{G}+\lambda_{f}$. 
The last statement, when $G$ is split, is proved in a similar manner using the commutative diagram

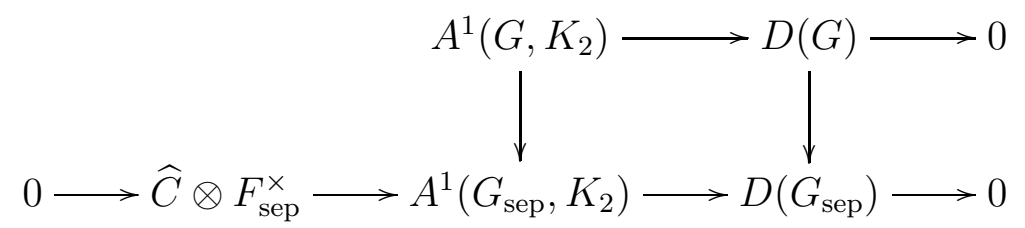

with the exact rows.

Now consider the map

$$
\varepsilon_{f}: D(G) \longrightarrow H^{2}(Y, \widehat{C}(1))
$$

for an arbitrary $G$-torsor $f: X \rightarrow Y$. We prove that $\varepsilon_{f}=\theta_{G}^{*}+\lambda_{f}^{*}$.

Choose a generically free representation $V$ of $G$ such that there is a $G$ equivariant open subset $U \subset V$ with the property $\operatorname{codim}_{V}(V \backslash U) \geq 2$ and a $G$-torsor $g: U \rightarrow Z$ with $Z(F) \neq \emptyset$ (see Section 5a). By assumption on codimension, we have $F[U]^{\times}=F^{\times}$and $\mathrm{CH}^{1}(U)=0$. It follows from (1.1) that $F[Z]^{\times}=F^{\times}$and $\mathrm{CH}^{1}(Z)=0$. By Corollary 6.4, the map

$$
H^{2}(Z, \widehat{C}(1)) \rightarrow H^{2}(F(Z), \widehat{C}(1))
$$

is injective.

Let $h: E \rightarrow$ Spec $F(Z)$ be the generic fiber of $g$. By Lemma 6.13 applied to the $G$-torsor $h, \varepsilon_{h}=\theta_{G}^{*}+\lambda_{h}^{*}$. Therefore,

$$
\left(\varepsilon_{g}\right)_{F(Z)}=\varepsilon_{h}=\theta_{G}^{*}+\lambda_{h}^{*}=\left(\theta_{G}^{*}+\lambda_{g}^{*}\right)_{F(Z)} .
$$

The injectivity of the map (6.10) implies that

$$
\varepsilon_{g}=\theta_{G}^{*}+\lambda_{g}^{*}
$$

Consider the $G$-torsor $k: X \times U \rightarrow W:=(X \times U) / G$. We have the natural projections $p: W \rightarrow Y$ and $W \rightarrow Z$. The inverse images of the $G$-torsors $f: X \rightarrow Y$ and $g: U \rightarrow Z$ to $W$ are both isomorphic to $k$. It follows that

$$
\left(\varepsilon_{f}\right)_{W}=\varepsilon_{k}=\left(\varepsilon_{g}\right)_{W} \quad \text { and } \quad\left(\lambda_{f}^{*}\right)_{W}=\lambda_{k}^{*}=\left(\lambda_{g}^{*}\right)_{W} .
$$

Note that $W$ is an open subvariety in the vector bundle $(X \times V) / G$ over $Y$ with the closed complement of codimension at least 2 . Hence by the homotopy invariance property for vector bundles, the natural maps $F\left[Y_{\text {sep }}\right]^{\times} \rightarrow$ $F\left[W_{\text {sep }}\right]^{\times}$and $\mathrm{CH}^{1}\left(Y_{\text {sep }}\right) \rightarrow \mathrm{CH}^{1}\left(W_{\text {sep }}\right)$ are isomorphisms. Moreover, $W$ is an open subset in the vector bundle $(X \times V) / G \rightarrow Y$, hence the field extension $F\left(W_{\text {sep }}\right) / F\left(Y_{\text {sep }}\right)$ is purely transcendental and therefore, the natural map $\operatorname{Br}\left(Y_{\text {sep }}\right) \rightarrow \operatorname{Br}\left(W_{\text {sep }}\right)$ is injective. By Lemma 6.5, the map $p^{*}: H^{2}(Y, \widehat{C}(1)) \rightarrow$ $H^{2}(W, \widehat{C}(1))$ is injective. By (6.11) and (6.12), we have

$$
\left(\varepsilon_{f}\right)_{W}=\varepsilon_{k}=\left(\varepsilon_{g}\right)_{W}=\left(\theta_{G}^{*}+\lambda_{g}^{*}\right)_{W}=\left(\theta_{G}^{*}+\lambda_{f}^{*}\right)_{W} .
$$

The injectivity of $p^{*}$ implies that $\varepsilon_{f}=\theta_{G}^{*}+\lambda_{f}^{*}$. 


\section{REFERENCES}

[1] S. Blinstein and A. Merkurjev, Cohomological invariants of algebraic tori, To appear in Algebra and Number Theory.

[2] A. Borel, Linear algebraic groups, second ed., Graduate Texts in Mathematics, vol. 126, Springer-Verlag, New York, 1991.

[3] D. Edidin and W. Graham, Equivariant intersection theory, Invent. Math. 131 (1998), no. 3, 595-634.

[4] H. Esnault, B. Kahn, M. Levine, and E. Viehweg, The Arason invariant and mod 2 algebraic cycles, J. Amer. Math. Soc. 11 (1998), no. 1, 73-118.

[5] R. Garibaldi, A. Merkurjev, and Serre J.-P., Cohomological invariants in Galois cohomology, American Mathematical Society, Providence, RI, 2003.

[6] B. Kahn, Applications of weight-two motivic cohomology, Doc. Math. 1 (1996), No. 17, $395-416$.

[7] M.-A. Knus, A. Merkurjev, M. Rost, and J.-P. Tignol, The book of involutions, American Mathematical Society, Providence, RI, 1998, With a preface in French by J. Tits.

[8] S. Lichtenbaum, The construction of weight-two arithmetic cohomology, Invent. Math. 88 (1987), no. 1, 183-215.

[9] S. Lichtenbaum, New results on weight-two motivic cohomology, The Grothendieck Festschrift, Vol. III, Birkhäuser Boston, Boston, MA, 1990, pp. 35-55.

[10] A. S. Merkurjev, Degree three cohomological invariants of semisimple groups, Preprint, http://www.math.ucla.edu/ merkurev/papers/ssinv2.pdf (2013).

[11] J. S. Milne, Étale cohomology, Princeton University Press, Princeton, N.J., 1980.

[12] D. Quillen, Higher algebraic K-theory. I, (1973), 85-147. Lecture Notes in Math., Vol. 341.

[13] M. Rost, Chow groups with coefficients, Doc. Math. 1 (1996), No. 16, 319-393 (electronic).

[14] J.-J. Sansuc, Groupe de Brauer et arithmétique des groupes algébriques linéaires sur un corps de nombres, J. Reine Angew. Math. 327 (1981), 12-80.

[15] A. A. Suslin, Torsion in $K_{2}$ of fields, K-Theory 1 (1987), no. 1, 5-29.

[16] J. Tits, Classification of algebraic semisimple groups, Algebraic Groups and Discontinuous Subgroups (Proc. Sympos. Pure Math., Boulder, Colo., 1965) (A. Borel and G. D. Mostow, eds.), vol. 9, Amer. Math. Soc., Providence, R.I., 1966, 1966, pp. 33-62.

Department of Mathematics, University of California, Los Angeles, CA 90095-1555, USA

E-mail address: merkurev@math.ucla.edu 